\title{
LCROSS (Lunar Crater Observation and Sensing Satellite) Observation Campaign: Strategies, Implementation, and Lessons Learned
}

Jennifer L. Heldmann • Anthony Colaprete • Diane H. Wooden •

Robert F. Ackermann • David D. Acton · Peter R. Backus • Vanessa Bailey • Jesse G. Ball • William C. Barott • Samantha K. Blair • Marc W. Buie • Shawn Callahan • Nancy J. Chanover • Young-Jun Choi • Al Conrad • Dolores M. Coulson • Kirk B. Crawford • Russell DeHart • Imke de Pater • Michael Disanti • James R. Forster • Reiko Furusho • Tetsuharu Fuse · Tom Geballe • J. Duane Gibson • David Goldstein • Stephen A. Gregory • David J. Gutierrez • Ryan T. Hamilton • Taiga Hamura • David E. Harker • Gerry R. Harp • Junichi Haruyama • Morag Hastie • Yutaka Hayano • Phillip Hinz • Peng K. Hong • Steven P. James • Toshihiko Kadono • Hideyo Kawakita • Michael S. Kelley • Daryl L. Kim • Kosuke Kurosawa • Duk-Hang Lee • Michael Long • Paul G. Lucey • Keith Marach • Anthony C. Matulonis • Richard M. McDermid • Russet McMillan • Charles Miller · Hong-Kyu Moon • Ryosuke Nakamura · Hirotomo Noda • Natsuko Okamura · Lawrence Ong • Dallan Porter • Jeffery J. Puschell • John T. Rayner · J. Jedadiah Rembold · Katherine C. Roth · Richard J. Rudy • Ray W. Russell · Eileen V. Ryan · William H. Ryan • Tomohiko Sekiguchi • Yasuhito Sekine • Mark A. Skinner • Mitsuru Sôma • Andrew W. Stephens • Alex Storrs • Robert M. Suggs • Seiji Sugita - Eon-Chang Sung • Naruhisa Takatoh • Jill C. Tarter · Scott M. Taylor · Hiroshi Terada • Chadwick J. Trujillo • Vidhya Vaitheeswaran • Faith Vilas • Brian D. Walls • Jun-ihi Watanabe • William J. Welch · Charles E. Woodward · Hong-Suh Yim • Eliot F. Young

Received: 9 October 2010 / Accepted: 8 February 2011 / Published online: 18 March 2011 (C) The Author(s) 2011. This article is published with open access at Springerlink.com

Abstract NASA's LCROSS (Lunar Crater Observation and Sensing Satellite) mission was designed to explore the nature of previously detected enhanced levels of hydrogen near the lunar poles. The LCROSS mission impacted the spent upper stage of the launch vehicle into

J.L. Heldmann $(\varangle) \cdot$ A. Colaprete $\cdot$ D.H. Wooden Division of Space Sciences and Astrobiology, NASA Ames Research Center, Moffett Field, CA, USA e-mail: Jennifer.Heldmann@nasa.gov

R.F. Ackermann · K.B. Crawford · D.J. Gutierrez · D.L. Kim · R.J. Rudy · R.W. Russell The Aerospace Corporation, Los Angeles, CA, USA

D.D. Acton · K. Marach · S.M. Taylor

Raytheon Vision Systems, Goleta, CA, USA

P.R. Backus - W.C. Barott · S.K. Blair · G.R. Harp · J.C. Tarter

SETI Institute, Mountain View, CA, USA 
a permanently shadowed region of the lunar surface to create an ejecta plume. The resultant impact crater and plume were then observed by the LCROSS Shepherding Spacecraft as well as a cadre of telescopes on the Earth and in space to determine the nature of the materials contained within the permanently shadowed region. The Shepherding Spacecraft then became a second impactor which was also observed by multiple assets.

The LCROSS Observation Campaign was a key component of the LCROSS mission. The goal of the Observation Campaign was to realize the scientific benefits of extending the LCROSS observations to multiple ground and space-based assets.

This paper describes the LCROSS Observation Campaign and provides an overview of the Campaign coordination and logistics as well as a summary of the observation techniques utilized at a multitude of observatories. Lessons learned from the LCROSS Observation Campaign are also discussed to assist with the planning of future unique observing events.

Keywords Moon $\cdot$ Missions $\cdot$ Observations $\cdot$ Telescopes

V. Bailey $\cdot$ P. Hinz $\cdot$ V. Vaitheeswaran

Steward Observatory, University of Arizona, Tucson, AZ, USA

J.G. Ball - D.M. Coulson · T. Geballe · A.C. Matulonis · R.M. McDermid · K.C. Roth · A.W. Stephens ·

C.J. Trujillo · B.D. Walls

Gemini Observatory, Hilo, HI, USA

M.W. Buie · E.F. Young

Southwest Research Institute, Boulder, CO, USA

S. Callahan · J.D. Gibson · M. Hastie · D. Porter · F. Vilas

MMT Observatory, Tucson, AZ, USA

N.J. Chanover · R.T. Hamilton · R. McMillan · C. Miller

Astronomy Department, New Mexico State University, Las Cruces, NM, USA

Y.-J. Choi · D.-H. Lee · H.-K. Moon · E.-C. Sung · H.-S. Yim

Korea Astronomy and Space Science Institute, Daejeon 305-348, South Korea

A. Conrad

Keck Observatory, Mauna Kea, HI, USA

R. DeHart

Honeywell Technology Solutions, Inc., Greenbelt, MD, USA

I. de Pater · J.R. Forster · W.J. Welch

UC Berkeley, 601 Campbell Hall, Berkeley, CA, USA

M. Disanti

NASA Goddard Space Flight Center, Greenbelt, MD, USA

R. Furusho $\cdot$ H. Noda $\cdot$ M. Sôma $\cdot$ J.-i. Watanabe

National Astronomical Observatory of Japan, Mitaka, Tokyo, Japan

T. Fuse $\cdot$ Y. Hayano $\cdot$ N. Takatoh $\cdot$ H. Terada

Subaru Telescope, National Astronomical Observatory of Japan, Hilo, HI, Japan

D. Goldstein

University of Texas, Austin, USA 


\section{Introduction}

\subsection{The LCROSS Mission}

The LCROSS mission was designed to explore a permanently shadowed region near either the lunar north or south pole of the Moon. Scientific goals of the mission included 1) to

S.A. Gregory - M.A. Skinner

Boeing LTS, Kihei, HI, USA

T. Hamura $\cdot$ P.K. Hong $\cdot$ K. Kurosawa $\cdot$ N. Okamura $\cdot$ Y. Sekine

Department of Complexity Science and Engineering, University of Tokyo, Kashiwa, Chiba, Japan

D.E. Harker

Center for Astronomy and Space Science, University of California, San Diego, CA, USA

J. Haruyama

Institute of Space and Astronautical Science, JAXA, Sagamihara, Kanagawa, Japan

S.P. James

Capt USAF, AFRL/RDSM Det 15, AF Maui Optical and Supercomputing Site (AMOS), Kihei, HI, USA

T. Kadono

Institute of Laser Engineering, Osaka University, Suita, Osaka, Japan

H. Kawakita

Department of Physics, Kyoto Sangyo University, Kita-ku, Kyoto, Japan

M.S. Kelley

Department of Astronomy, University of Maryland, College Park, MD, USA

D.-H. Lee

University of Science and Technology, Daejeon 305-350, South Korea

M. Long

Premiere Wireless, Orange, CA, USA

P.G. Lucey

Hawaii Institute of Geophysics and Planetology, University of Hawaii, Honolulu, HI, USA

R. McMillan

Apache Point Observatory, Sunspot, NM, USA

R. Nakamura

National Institute of Advanced Industrial Science and Technology, Tsukuba, Ibaraki, Japan

L. Ong

NASA Goddard/Science Systems and Applications, Inc., Greenbelt, MD, USA

J.J. Puschell

Raytheon Space and Airborne Systems, El Segundo, CA, USA

J.T. Rayner

Institute for Astronomy, University of Hawaii, Hilo, HI, USA

J.J. Rembold · E.V. Ryan · W.H. Ryan

New Mexico Tech, Socorro, NM, USA 
identify the form of the hydrogen observed at the lunar poles, 2) to quantify, if present, the amount of water in the lunar regolith with respect to hydrogen concentrations, and 3) to characterize the lunar regolith within a permanently shadowed region on the Moon. LCROSS also provided a unique experiment to understand the anatomy of a lunar impact by planning the impact at a known time and location on the Moon which enabled unprecedented observations of the event.

LCROSS was designed to explore a unique permanently shadowed region of the lunar south pole. Due to the low angle between the Moon's rotational axis and the normal to the ecliptic plane combined with the low inclination of the Earth's orbit around the Sun, and the lunar topography, permanently shadowed regions exist at both poles of the Moon and are among the coldest regions in the Solar System (Arnold 1979; Bussey et al. 2003; Feldman et al. 2001; Crider and Vondrak 2003; Margot et al. 1999; Stacy et al. 1997; Vasavada et al. 1999). The temperature of the floor of the Cabeus Crater at the impact site has been measured at $45 \mathrm{~K}$ by the Diviner instrument aboard the Lunar Reconnaissance Orbiter (LRO) (Paige et al. 2010). The low temperatures of these permanently shadowed regions make these areas prime candidates for cold traps where volatile species can be stable for billions of years (Vasavada et al. 1999).

Due to this unique environment capable of trapping volatile species, the permanently shadowed regions of the lunar poles are especially intriguing scientifically. However, these areas have been unexplored in situ because the permanent darkness coupled with the extremely low temperatures presents significant engineering challenges for both robotic and human explorers. Previous landed missions have thus been largely confined to equatorial regions where sunlight illuminates the surface for $\sim 2$ weeks of daylight each month. The lunar poles have never been explored in situ prior to the LCROSS mission. Previous lunar orbital missions have been unable to gather meaningful data in the UV-visible portion of the spectrum because direct sunlight never reaches these shadowed regions and studies have thus been largely based on radar data (Nozette et al. 1996; Margot et al. 1999) and neutron spectroscopy (Feldman et al. 1998, 2001) which are tantalizing but inconclusive. By bringing material into sunlight, LCROSS has therefore provided an unprecedented opportunity to understand the characteristics of this heretofore unexplored permanently shadowed polar terrain.

Objects impact the Moon regularly and are routinely observed via a program established by NASA's Meteoroid Environment Office. Earth-based observations of the dark portion of the Moon can detect impacts of meteroids greater than 500 grams which impact the lunar surface (Suggs et al. 2008a). The Moon is typically monitored for an average of 10 nights per month with $0.25,0.36$ and $0.5 \mathrm{~m}$ telescopes and low-light-level video cameras, and

T. Sekiguchi

Hokkaido University of Education, Kita-ku, Sapporo, Japan

A. Storrs

Towson University, Towson, MD, USA

R.M. Suggs

NASA Marshall Space Flight Center, Huntsville, AL, USA

S. Sugita

University of Tokyo, Tokyo, Japan

C.E. Woodward

Department of Astronomy, University of Minnesota, Minneapolis, MN, USA 
flashes indicative of a lunar impact are positively identified by two separate telescopes for confirmation of the impact event. Over 200 such impacts have been observed over a 2-year period (Suggs et al. 2008b). A prime difference between these naturally occurring lunar impacts from meteoroids and the LCROSS mission is that the exact time and location of impact was known with great precision for LCROSS. The availability of this information enabled the planning of observations specifically tailored to collecting data of the impact event (Heldmann et al. 2008, 2009). In addition, the timing of the LCROSS impacts as well as the lunar impact site were chosen specifically to enable data collection from various observing assets. Therefore observations from the Moon (Lunar Reconnaissance Orbiter and LCROSS), Earth-orbit, and ground-based observing assets were all trained on the LCROSS impact site on the night of impact (Heldmann et al. 2009).

\subsection{LCROSS Mission Architecture}

The LCROSS spacecraft was launched as a comanifested payload with the Lunar Reconnaissance Orbiter (LRO). The LCROSS mission used the Atlas V Centaur Earth departure upper stage of the launch vehicle as a $2,366 \mathrm{~kg}$ kinetic impactor. The impact created an ejecta plume whose properties were observed by a Shepherding Spacecraft $(\mathrm{S}-\mathrm{S} / \mathrm{C})$ plus Earth- and space-based telescopes. Following a similar trajectory of the Centaur, the $625 \mathrm{~kg}$ $\mathrm{S}-\mathrm{S} / \mathrm{C}$ flew through the Centaur impact plume before impacting the Moon itself. LCROSS was developed and managed by NASA Ames Research Center in partnership with Northrop Grumman.

The launch of both LRO and LCROSS occurred from Cape Canaveral Air Force Station onboard an Atlas V rocket on June 18, 2009. After launch, the LRO spacecraft separated from LCROSS and the launch vehicle as planned and traveled to the Moon to enter lunar orbit and complete its mission objectives (Vondrak et al. 2010). The LCROSS spacecraft remained attached to the Centaur upper stage of the launch vehicle and completed a swingby of the Moon on Day 5 after launch. The LCROSS payload was successfully tested and all instruments responded nominally to gather data when pointed at several different locations on the Moon. LCROSS and the attached Centaur then entered a large Lunar Gravity-Assist, Lunar Return Orbit (LGALRO) orbit around the Earth (Fig. 1). LCROSS completed three LGALRO orbits each with a $\sim 36$ day period. The LGALRO orbit period served several

\section{LCROSS Orbit - From Ecliptic North}

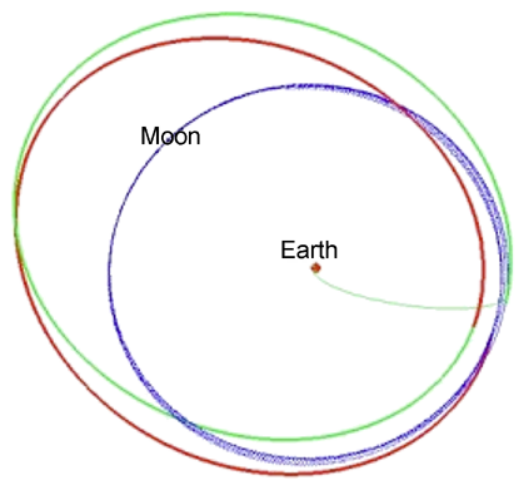

LCROSS Orbit - Side View

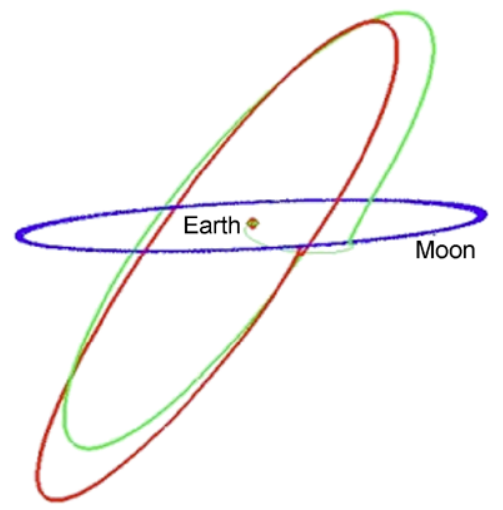

Fig. 1 The LCROSS Lunar Gravity-Assist, Lunar Return Orbit (LGALRO) orbit 
purposes as it 1) allowed for accurate targeting of the lunar impact site, 2) provided LRO with sufficient time to enter lunar orbit, complete on-orbit check-outs, and acquire data to assist with final LCROSS site selection, and 3) provided time in flight to bake out and de-gas the Centaur in order to minimize any risk of contamination upon lunar impact.

At nine hours fifty minutes before lunar impact the Shepherding Spacecraft and Centaur rocket stage successfully separated. The Shepherding Spacecraft then completed a 180degree flip to orient the Payload Observation Deck (POD) towards the Moon. With this maneuver, the POD was facing the Moon and in the proper configuration to allow the spacecraft instruments to gather data while observing the Moon and Centaur impact. A braking burn was performed to introduce a four-minute separation between the Centaur and Shepherding Spacecraft. This separation between the Centaur and Shepherding Spacecraft was required in order to provide the Shepherding Spacecraft payload instruments with the optimal viewing conditions of the Centaur impact. The instruments were turned on and collecting data for the final hour before impact of the S-S/C, although the critical science phase was the final four minutes which is the period during and after the Centaur impact.

At 11:31:19.506 UTC on 9 October 2009 the spent Centaur rocket stage impacted the lunar surface within the Cabeus Crater at 84.675 degrees South latitude and -48.703 degrees East longitude. An image of the impact site obtained from the visible camera aboard the S-S/C is shown in Fig. 2. At 11:35:36.116 UTC on 9 October 2009 the Shepherding Spacecraft impacted the Moon at 84.719 degrees South, -49.610 degrees East longitude. The physical separation of the Centaur and S-S/C impacts was $2.857 \mathrm{~km}$. Data were successfully collected by the instruments aboard the Shepherding Spacecraft up until S-S/C impact and loss of signal from the Shepherding Spacecraft.

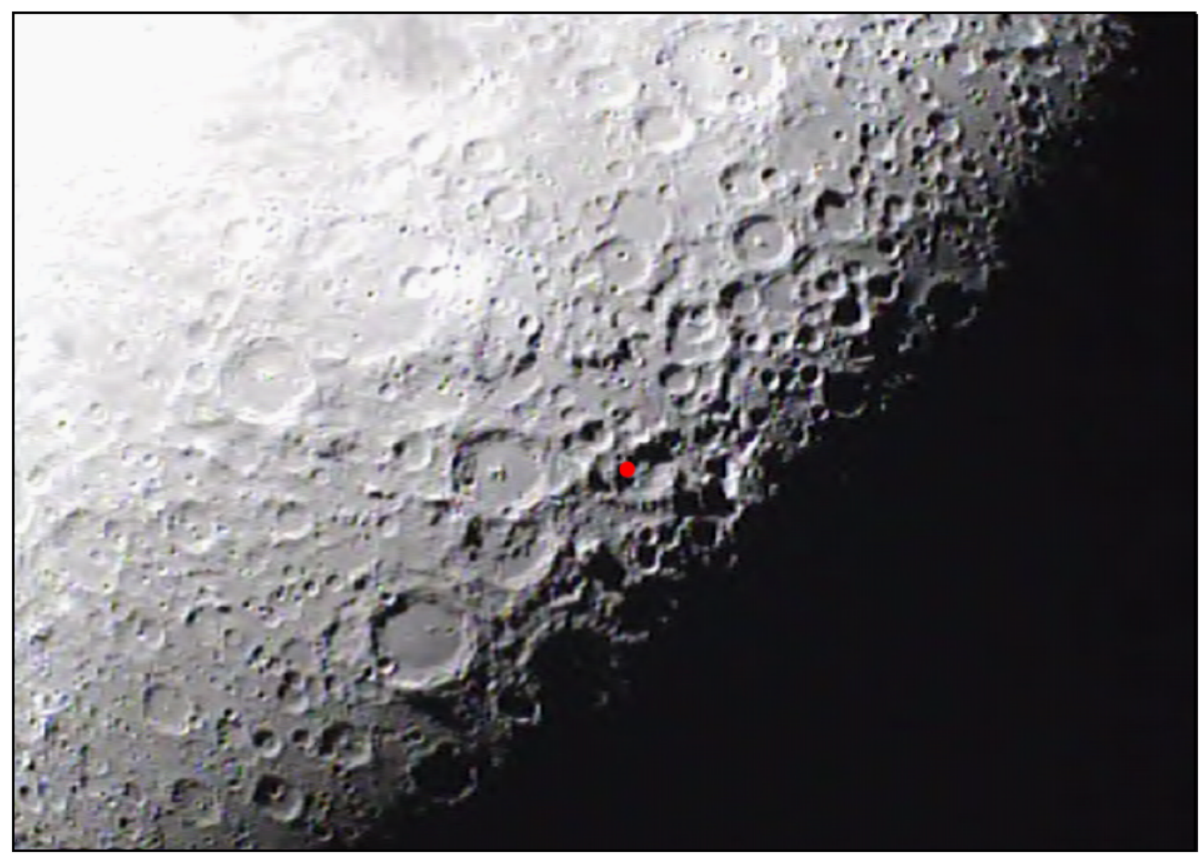

Fig. 2 LCROSS Visible Camera image of the Moon acquired by the Shepherding Spacecraft. The LCROSS impact site of the Centaur and Shepherding Spacecraft in Cabeus Crater is encompassed within the red dot 
Fig. 3 (a) View of the Moon from Earth on the date of the LCROSS impact (9 October 2009). Earth-Observing-1 (EO-1) satellite pre-impact image.

(b) Terrestrial landmasses facing the Moon at the time of impact

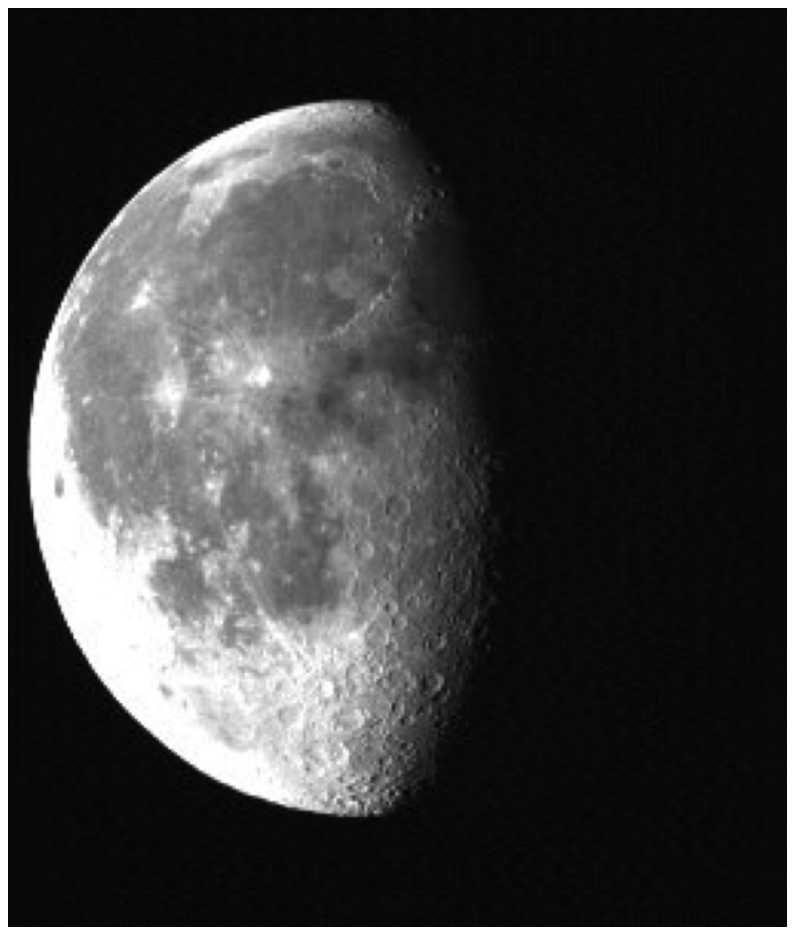

Observations of the Centaur impact plume from the Shepherding Spacecraft, groundbased, and space-based observing assets were possible primarily after the ejecta had traveled high enough above the lunar surface to reach sunlight. The sun horizon was $\sim 833$ meters above the lunar surface at the Cabeus impact site, and therefore the reflected light from the lofted particles was only observable once the ejecta had reached this altitude. The Shepherding Spacecraft impact would only be observable by the ground-based and space-based assets. Similar to the Centaur impact, the S-S/C impact ejecta also had to travel up into sunlight to enable most of these observations. Figure 3a shows the phase of the Moon at the time of impact. The Moon was visible from the western hemisphere of Earth (Fig. 3b) meaning that these terrestrial landmasses had a direct line-of-sight to observe the impact events.

\subsection{LCROSS Impact Site}

The LCROSS impact was purposely designed to provide maximum observational coverage from a variety of observing assets in order to collect the most data possible to address the science and exploration objectives of the mission. Below we discuss several key parameters that influenced the observability of the impacts from ground and space-based assets.

In addition, the LCROSS impact site was chosen to maximize the probability of addressing the mission's science and exploration goals. Below multiple target selection criterion are described (not in priority order).

\subsubsection{Permanent Shadow}

LCROSS was required to target a 10-km radius impact area (3 sigma) and impact in a region of permanent shadow poleward of 70 degrees latitude at either lunar pole. Based on previous 


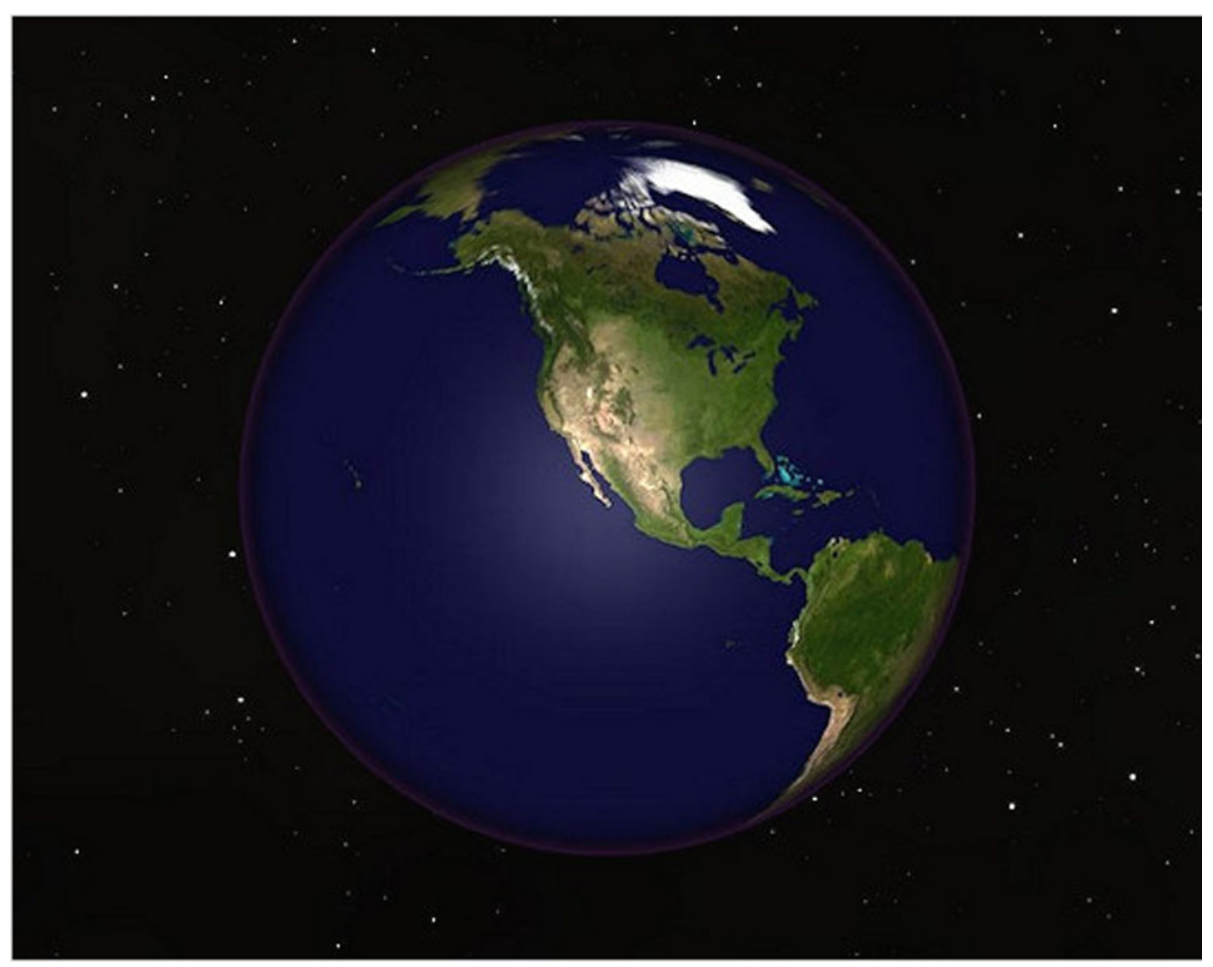

Fig. 3 (Continued)

mission data, the lunar poles could harbor volatiles (Crider and Vondrak 2003, Feldman et al. 1998, 2001; Lawrence et al. 2006; Nozette et al. 1996; Vasavada et al. 1999).

\subsubsection{Topographic Mask}

The topographic mask is the vertical extent of the shadowed region extending from the lunar surface to the Sun horizon. This height was targeted to be $<3 \mathrm{~km}$ (required) with a goal of $<1 \mathrm{~km}$. This height from impact site to sunlight is important because most observations of the impact plume are only enabled when the plume reaches sunlight and light is reflected off of plume dust and/or ice particles. Also, less ejecta reaches higher altitudes, so a lower topographic mask is advantageous to observers such that more ejecta reaches the sunlight yielding more flux and making observations easier. The ability to observe from Earth is a minimum requirement and provides complimentary data (e.g., more than just backup data to the LCROSS S-S/C observations). Note that spacecraft data were collected during the impact flash period which does not require ejecta illumination. However, Earth-based observations were enabled when the ejected material rises above the crater rim into the sunlight.

\subsubsection{Association with Increased Hydrogen Concentrations}

LCROSS had a goal to impact in a location where the measured hydrogen was consistent with Water Equivalent Hydrogen (WEH) levels of greater than $0.5 \%$ (the LCROSS Spacecraft detection limit requirement). Site selection analysis utilized two datasets to assess the 
hydrogen levels, namely Lunar Prospector neutron data with the Pixon Recovery analysis and LRO Lunar Exploration Neutron Detector (LEND) observations.

\subsubsection{Flat and Smooth Terrain}

LCROSS targeted slopes across 500 meters (100 meter goal) baseline with slopes $<20$ degrees required and $<15$ degrees desired. Minimal surface roughness requirements were set at less than one 10 meter diameter rock per square $\mathrm{km}$ and less than 300 five meter diameter rocks per square $\mathrm{km}$. The goal of these requirements was to impact in a region relatively free from surface rocks and boulders in order to impact and explore the regolith material which may be harboring volatiles. The impact ejecta "productivity" is dependent on slope and roughness effects. Impacts into slopes greater than 20 degrees across areas larger than the crater scale can direct ejecta laterally reducing the total ejecta at altitude. Roughness (or blockiness) of the surface can also affect the total ejecta excavated and its velocity distribution. Impacting into a rough or blocky terrain will cause the energy to go into the crushing of rock or inter-block slipping/compaction rather than "simple fluid" motion. Impacting into a smoother terrain instead of hilly or undulating terrain also helps to maximize the amount of ejecta material reaching the sunlight for observation. The LCROSS Project used radar, altimetry, LOLA (Lunar Orbiter Laser Altimeter) pulse width and imaging (Kaguya Terrain Camera and LRO LROC (Lunar Reconnaissance Orbiter Camera)) to characterize slopes (quantitative) and roughness (qualitative).

\subsubsection{Earth View}

LCROSS targeted an impact site where the ejecta plume would be within direct line-of-sight to Earth. The LCROSS impact site was specifically selected to not preclude observation of the impact from Earth-based assets. Impacting on the near-side of the Moon was required to enable Earth-based observations of the impact. Site selection also considered additional factors critical to Earth-based observing. For example, the scene from Earth was considered, e.g., whether the expected ejecta contrast would be against a lit Moon or shadow background, and the ability to locate the target. To maximize the effectiveness of the observation support, the LCROSS project aimed to have the impacts occur at a time of month at least 30 degrees away from full or new Moon. Because of the concentration of professional observatories that planned to observe the impacts from Hawaii, the LCROSS Project aimed to have the impact occur at least two (2) hours after dusk and two (2) hours prior to dawn and have the Moon at least 45 degrees above the horizon in Hawaii. These constraints allowed telescopes as far east as Massachusetts to observe the impact.

Impact site characterization began prior to LCROSS selection (as part of the proposal process) and continued until several weeks prior to impact. Both our understanding of the impact processes as well as observation of the impact and the targets matured considerably during this timeframe. New data from the Kaguya, Chandrayaan-1, and LRO spacecraft were critical in the site selection process. Taking these criteria into account, along with the orbital dynamics dictated by launch date regarding the accessibility of the sites with the spacecraft, the target list of candidates evolved with time.

Ultimately, LCROSS impacted into Cabeus Crater. Cabeus was observable from Earth, had a significant amount of hydrogen, showed a relatively flat and non-blocky floor, and had a reasonable topographic mask. We note that the height to sunlight from the impact site was 833 meters but due to the presence of a hill in the line-of-sight from Earth, ejecta would have to travel vertically on the order of $2.5 \mathrm{~km}$ in height to be observable from Earth. 


\subsection{Anatomy of the Impact}

The impact event can be divided into the flash, curtain, and crater phases as shown in Fig. 4. Each of these phases is summarized below. This information was provided to the Astronomers to facilitate the planning of observations.

Flash At impact, the kinetic energy of the projectile is transferred to the kinetic (ejecta) and internal energy of the target (compaction, heating). A portion of the internal energy may induce vapor resulting in vibrational and rotational emission lines that will evolve with space and time. The intensity and decay of the initial flash are related to the physical structure of the target (porosity, strength, volatile content, composition). Consequently, characterization of the initial flash provides a complementary tool to understand initial coupling and the nature of the target. The flash phase is expected to last less than one second. Since the Cabeus impact location was not within line-of-sight to Earth, the flash was not observable to ground-based telescopes.

Curtain Curtain phase refers to the time after impact when ejecta has been lofted above the lunar surface. Once the ejecta reaches sunlight then the ejecta are illuminated via reflected sunlight. Ballistic debris forms the ejecta curtain, and any water lofted into sunlight may begin to photodissociate. The curtain phase is expected to last on the order of several minutes.

Crater Crater phase refers to the production of the crater created upon impact of the Centaur on the lunar surface. The curtain phase is nearly (if not fully) complete and the ejecta has returned to the lunar surface. The crater phase can last for several hours as the lunar surface returns to thermal equilibrium following the impact event and the $\mathrm{OH}$ exosphere created by the release of water ice and/or vapor into sunlight dissipates.

\subsection{Impact Model Predictions}

The LCROSS mission used the impact of the Atlas V Centaur upper stage to excavate and eject lunar surface material to a location where it could be observed by both the LCROSS $\mathrm{S}-\mathrm{S} / \mathrm{C}$ and other lunar orbiting, earth orbiting and ground based assets. The S-S/C itself also impacted the lunar surface and created a second debris plume. It was necessary to model the expected results of the impact in order to plan and deploy the most effective observational campaign.
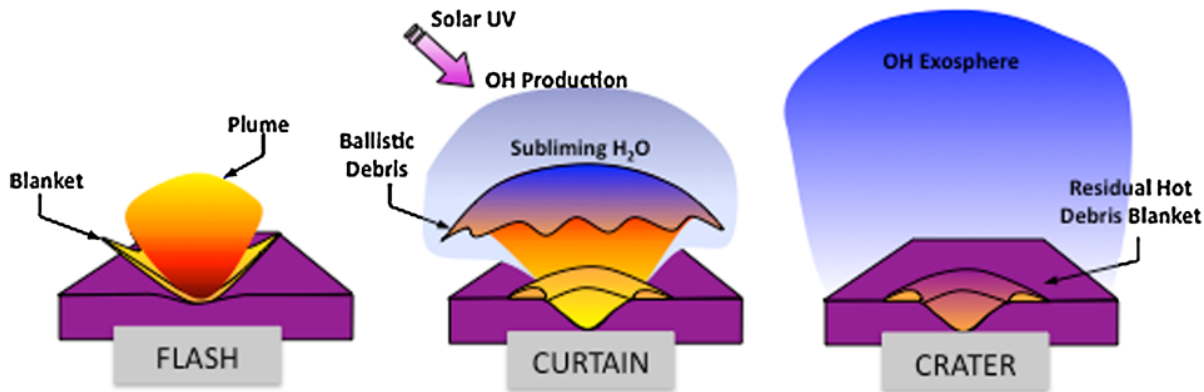

Fig. 4 The three stages of the LCROSS impact including Flash, Curtain, and Crater 
Both the Centaur and S-S/C impacts occurred at velocities of about $2.5 \mathrm{~km} / \mathrm{s}$ and thus are considered low-velocity impacts compared to natural impacts of meteors with velocities greater than $25 \mathrm{~km} / \mathrm{s}$. Prior to the lunar impact, the expected resulting crater diameter from the primary Centaur impact was approximately 18 meters. This crater diameter was the best estimate crater diameter based on a variety of independent efforts that included empirical scaling relations, analytic and numerical modeling. Using this crater diameter and a Monte Carlo model of the distribution of expected ejecta, a conservative upper estimate for the total displaced mass from the primary Centaur impact was approximately 500,000 kg (Korycansky et al. 2009). This value represents the total displaced mass, not the total mass that is thrown upward out of the crater. The amount of this material that is thrown upward depends on the velocity distribution of the ejecta, which in turn depends on a variety of other factors including the mass-velocity distribution exponent and ejecta flight angle. A series of Monte Carlo simulations was performed to place limits on the total ejecta mass exceeding an altitude above the lunar surface (Korycansky et al. 2009). Table 1 lists the amount of ejecta above a certain velocity taken from these Monte Carlo runs. Higher velocities are required for the ejecta to reach higher altitudes above the lunar surface. For example, velocities greater than about $250 \mathrm{~m} / \mathrm{s}$ are required to reach altitudes of greater than $35 \mathrm{~km}$. On average, in the Monte Carlo runs, only $0.35 \%$ of the total ejecta have velocities in excess of $250 \mathrm{~m} / \mathrm{s}$. Less than $0.01 \%$ of the mass has velocities greater than the escape velocity $(\sim 2.2 \mathrm{~km} / \mathrm{s})$ of the Moon (note: all velocities in the Monte Carlo simulations are calculated from scaling laws Housen et al. 1983 derived for faster (>10 km/s) moving impacts and should be considered upper bounds as these relationships are known to over-estimate the amount of material moving faster than about $250 \mathrm{~m} / \mathrm{s}$ ).

Figure 5 shows the predicted flight path (altitude versus time) for material with a particular velocity and the mass weighted ejecta altitude (i.e., the altitude at which the most mass exists). As is evident by the mass weighted ejecta altitude, only very small amounts of material reach altitudes above $10 \mathrm{~km}$. Also shown in Fig. 5 is the radial extent of the Centaur ejecta cloud as a function of time after impact. The ejecta radius was estimated analytically by setting the ejecta trajectory altitude to zero and solving for the curtain position. This is technically the "inner edge" of the ejecta curtain, as the outer edge is a monotonically decreasing function with distance. However, experiments have shown that this inner edge is a reasonable marker of the overall lateral extent of the curtain and the outer edge to be fairly well confined in extent and width that is proportional to the transient crater size. (It should be noted that these model predictions represent an idealized representation of the shape and low density of the impactor. Modeling of the impact using the complex internal structure of the actual Centaur is not currently feasible given limitations in computational power and

Table 1 Fraction (\%) of ejecta moving faster than velocity $v$

\begin{tabular}{lcccccc}
\hline & Min & $10 \%$ & Med & Avg. & $90 \%$ & Max \\
\hline$m\left(v>10 \mathrm{~m} \mathrm{~s}^{-1}\right)$ & 10.6 & 15.2 & 23.6 & 24.3 & 34.5 & 41.8 \\
$m\left(v>50 \mathrm{~m} \mathrm{~s}^{-1}\right)$ & 0.43 & 0.74 & 2.21 & 2.78 & 5.85 & 8.21 \\
$m\left(v>100 \mathrm{~m} \mathrm{~s}^{-1}\right)$ & 0.11 & 0.20 & 0.79 & 1.14 & 2.71 & 4.02 \\
$m\left(v>250 \mathrm{~m} \mathrm{~s}^{-1}\right)$ & 0.017 & 0.035 & 0.20 & 0.35 & 0.94 & 1.50 \\
$m\left(v>450 \mathrm{~m} \mathrm{~s}^{-1}\right)$ & 0.0052 & 0.011 & 0.080 & 0.16 & 0.46 & 0.76 \\
$m\left(v>800 \mathrm{~m} \mathrm{~s}^{-1}\right)$ & 0.0015 & 0.0034 & 0.030 & 0.068 & 0.20 & 0.35 \\
$m\left(v>1800 \mathrm{~m} \mathrm{~s}^{-1}\right)$ & 0.00014 & 0.00035 & 0.0038 & 0.010 & 0.31 & 0.056 \\
\hline
\end{tabular}


Fig. 5 The predicted altitude for material with velocity as indicated in the figure and the mass weighted ejecta altitude as a function of time after Centaur impact; Right axis: The predicted ejecta curtain radius as a function of time after Centaur impact

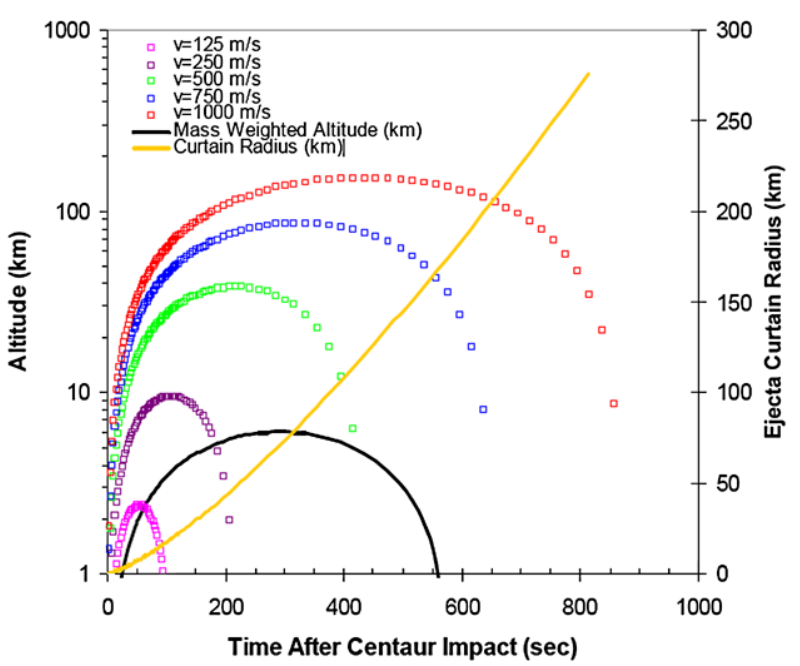

model capabilities. Estimates of the total displaced mass will likely be reduced from this idealized representation as modeling efforts become more robust).

As seen in Fig. 5, most material (by mass) will have returned to the surface after approximately 550 seconds ( 9.2 minutes) and has traveled laterally approximately $150 \mathrm{~km}$. In the first 200 seconds following impact, when material densities are at their maximum, the ejecta curtain radius will be approximately $35 \mathrm{~km}(\sim 1$ degree of latitude). All debris will be on ballistic trajectories originating from the lunar surface and without an additional perturbation to these trajectories will return to the lunar surface.

From ejecta trajectory analysis, total water and water opacity versus time were estimated as a function of material above an altitude (the altitude representing topographic mask height). The LCROSS spacecraft water sensitivity versus ejecta altitude calculations were required to meet $0.5 \%$ detection requirement with a solar topography mask $<3 \mathrm{~km}$ (Fig. 6).

\subsection{Note on Contamination}

After launch the Centaur stage of the Atlas V rocket contained some water or hydrogen and oxygen bearing compounds that could constitute contamination to the actual hydrogen signal trying to be measured. It is important to understand the contributions from these contaminants in the interpretation of all data from the Spacecraft and Observation Campaign. This contamination on the Centaur stage is in the form of liquid hydrogen and oxygen, hydrazine, and water, in batteries and as part of adhesives, potting materials, etc. (It must be noted that the presence of hydrogen does not necessarily translate to a contaminant. The measurement techniques to be employed by the LCROSS Spacecraft and Observation Campaign assets are not sensitive to hydrogen by itself, but rather compounds that contain hydrogen.) To minimize the effect of water contamination that may be present in the impacting Centaur several measures were taken during flight:

1. Liquid hydrogen and oxygen fuel remaining after the lunar transit burn were vented.

2. Remaining hydrazine fuel was vented.

3. The launch vehicle provider made an estimate of all water or water/hydrogen bearing materials. 


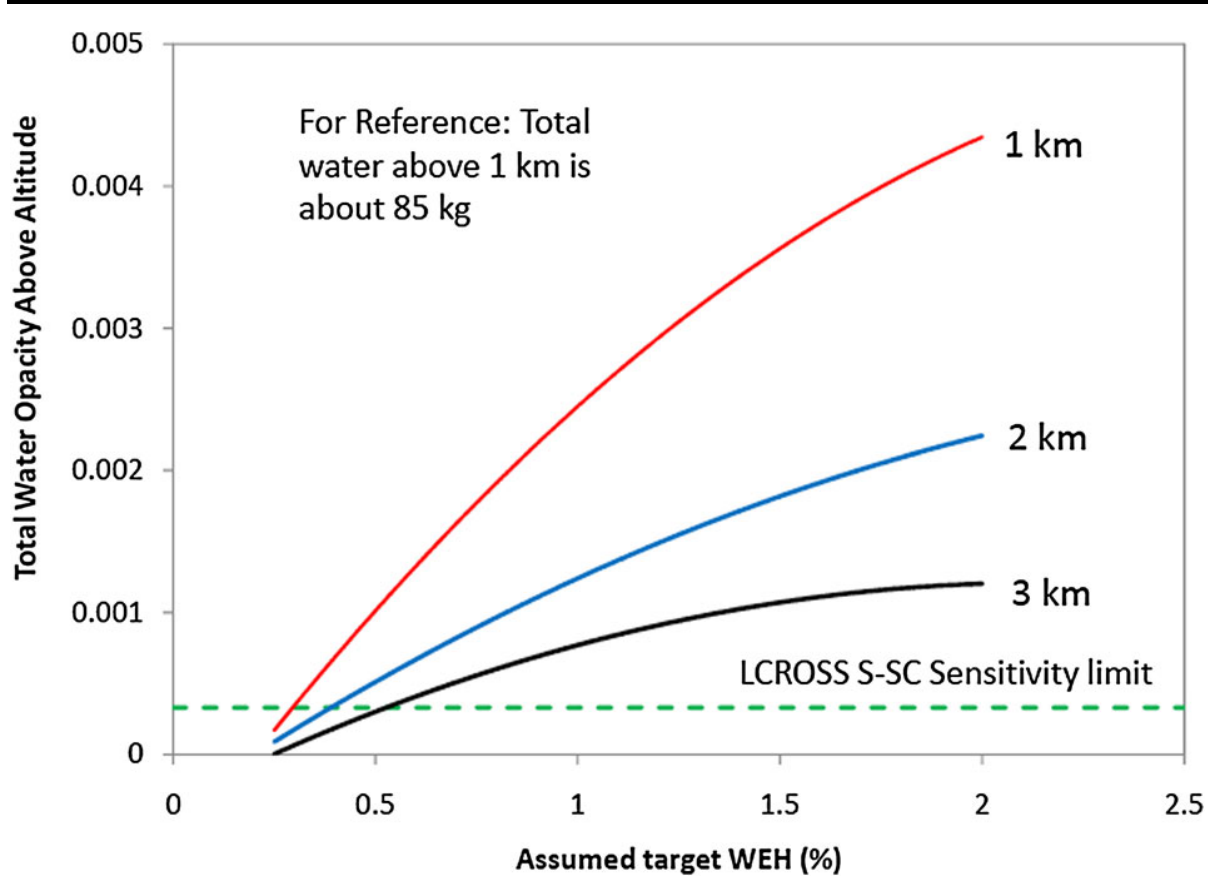

Fig. 6 LCROSS Spacecraft water sensitivity as a function of ejecta altitude

4. The total combined hydrogen and oxygen mass was required to be kept below $100 \mathrm{~kg}$ (corresponding to the maximum amount allowed to keep the contamination level to $0.1 \%$ within the ejecta curtain, assuming a conservative total ejected mass of $200,000 \mathrm{~kg}$ ).

The final contamination values at the time of impact were as follows: $\mathrm{O}_{2}(<0.5 \mathrm{~kg}), \mathrm{H}_{2}$ $(<0.02 \mathrm{~kg})$, water ice $(<0.51 \mathrm{~kg})$, water $(<0.12 \mathrm{~kg}), \mathrm{OH}$ as battery electrolyte $(<5.6 \mathrm{~kg})$, $\mathrm{N} 2 \mathrm{H} 4$ hydrazine fuel $(<0.05 \mathrm{~kg})$.

\section{Observation Campaign}

\subsection{Observation Campaign Overview}

The LCROSS Observation Campaign was a key component of the LCROSS mission ever since mission inception. The goal of the Observation Campaign was to realize the scientific benefits of extending the LCROSS observations to multiple ground and space-based observing assets. These observing assets provided a different vantage point compared to the LCROSS Spacecraft and therefore were able to observe the impact plume from different angles to provide a more comprehensive understanding of the plume formation and evolution. These additional observing assets also provided access to a variety of different instrumentation that could collect complimentary datasets. The variety of types of data (imagery, spectroscopy, etc.) coupled with the different wavelength regimes covered can provide a more comprehensive summary of the plume dynamics and enhance our scientific understanding of the lunar cold traps. In addition to the myriad of scientific benefits of multiple observations from a variety of observing platforms, the Observation Campaign also provided a contin- 
gency backup for observations in the event that the Shepherding Spacecraft was unable to observe the plume and/or to return the instrument data to Earth.

The LCROSS Project actively sought to engage the astronomical community early in the Observation Campaign process through a variety of different means. The Observation Campaign Coordinator (OCC) made multiple presentations at a variety of professional meetings to advertise the LCROSS observing opportunity and encourage Astronomers to participate in the Observation Campaign. Specifically, presentations were made at the Lunar and Planetary Science Conference (LPSC), American Astronomical Society Division for Planetary Sciences (DPS) meeting, American Astronomical Society (AAS), American Geophysical Union (AGU), Lunar Exploration Analysis Group (LEAG), and NASA Lunar Science Institute Lunar Science Forum (LSF). In addition, the OCC contacted numerous professional observatories directly to explain the unique LCROSS mission and observing opportunity. Due to the nature of the mission profile, observing time was required on one specific night with no flexibility to move an observing run to a different time. This constraint posed non-trivial challenges in terms of telescope time scheduling, and the LCROSS Project thus sought to provide the observatories with this information in support of impending observing proposals from Astronomer teams. The LCROSS Project also responded to inquiries from observatories when queried regarding the unique nature of the impacts to facilitate with the planning of observations, and provided letters of support where requested.

To realize the objectives of the Observation Campaign, the LCROSS professional Astronomers were included as official members of the LCROSS Science Team. Including the Astronomers as official members enabled the LCROSS Project to share mission critical information with the observing teams and disseminate this information to inform and enable the planning of observations. For example, throughout the site selection process, Astronomers were informed and consulted on impact site selection decisions. The impact site was important for observers in terms of terrain illumination (e.g., the impact plume could be superimposed on either a black (shadowed) or sunlit terrain depending on impact site) and pointing considerations (many Astronomers conducted practice observing runs to become familiar with the local lunar topography to enable accurate pointing at the impact location). For example, visualizations such as shown in Fig. 7 were provided to the Astronomers to show the view of the south pole limb as seen from Earth and indicate the expected location of the impact plume. Impact plume predictions generated by the LCROSS Science Team were routinely shared with the Astronomers. Information such as expected plume brightness, size, and duration was important for Astronomer planning of observations, selection of appropriate instrumentation, determining exposure times, deciding to use sub-arrays on detectors, etc. The LCROSS impact date and time were dictated by the launch date as well as impact site, and the Astronomers were kept up-to-date on this topic as the scheduling of telescope time at various observatories is a non-trivial matter and the most timely information on impact time and date was essential for securing observing time on the night of impact. This mode of operation in which mission information was shared with the Astronomers was essential to the Observation Campaign as data such as lunar impact site, impact plume predictions, impact date and time, etc. were all critical parameters required by the Astronomers and observatories to enable the most robust set of observations from each site.

Information was shared between the LCROSS Science Team and the Astronomer teams through a variety of different communication mechanisms. The main point of contact for this purpose from the LCROSS Science Team was the LCROSS Observation Campaign Coordinator (OCC). The LCROSS OCC was also a member of the LCROSS Science and Payload teams and thus was intimately familiar with mission activities. The OCC was responsible for filtering the mission information and providing updates to the Astronomer teams on the data 


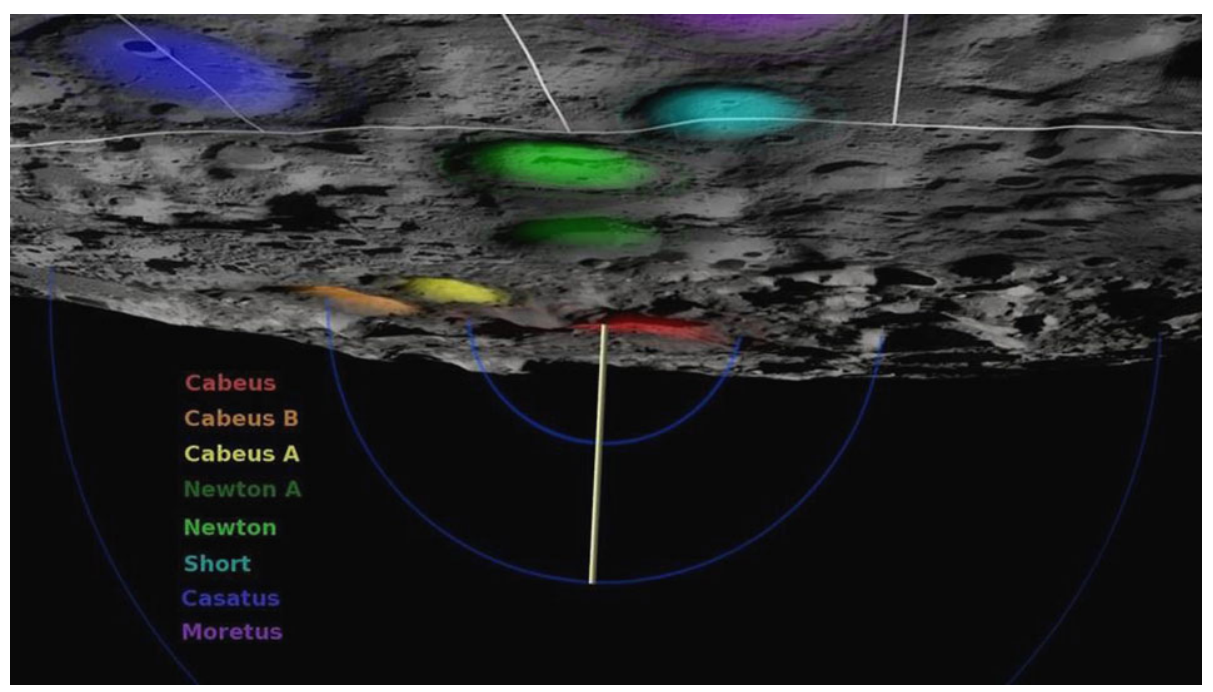

Fig. 7 This visualization of the lunar south pole limb shows the view from Mauna Kea, Hawaii on 9 October 2009 at 11:30 UT. Craters are labeled in color. The vertical white line is a $100 \mathrm{~km}$ flagpole and shows the expected location of the impact plume. The blue arcs show distances of $50 \mathrm{~km}, 100 \mathrm{~km}$, and $200 \mathrm{~km}$ from the lunar surface. Image courtesy of NASA Goddard Space Flight Center Scientific Visualization Studio

most relevant for the planning and execution of the Astronomer observations. The LCROSS OCC also responded to requests for specific information from the observing teams, and ensured a two-way dialog between the LCROSS Project and the Astronomers. Communications were primarily via email, although information was also exchanged via teleconferences, online conferencing, and at the LCROSS Astronomer Workshop held at NASA Ames Research Center in February 2008. In addition, a secure online repository for data and information files was hosted by NASA Ames which required a username and password to allow access to files to known participants within the LCROSS Observation Campaign.

After data collection by each observatory on impact night, the intent of the LCROSS Observation Campaign was to continue the collaborations amongst the various observing teams. Each team at each observatory had planned on acquiring a unique dataset spanning wavelength regimes from the ultraviolet to the radio portions of the spectrum (as described later in this paper). Based on experiences with previous observation campaigns such as occurred with the Deep Impact mission (Meech et al. 2005), the maximum science information would be gained by analyzing these complimentary datasets together. In addition, the LCROSS Project was poised to share the data and results from the S-S/C observations to assist with the interpretation of the ground-based and space-based assets. Each observing team reserved the right to analyze their own data but would benefit from cross-correlations and discussions with other scientists. Data sharing was made possible through the use of the secure online repository hosted by NASA Ames. In addition, discussions were facilitated through post-impact teleconferences, email, and phone conversations.

\subsection{Impact Night Observation Campaign Logistics}

Communication from the LCROSS Project to the Astronomers at the various observatories was critical during the mission to provide real-time updates needed for accurate observation. Here we describe the communications architecture and timeline for data transmission on the 


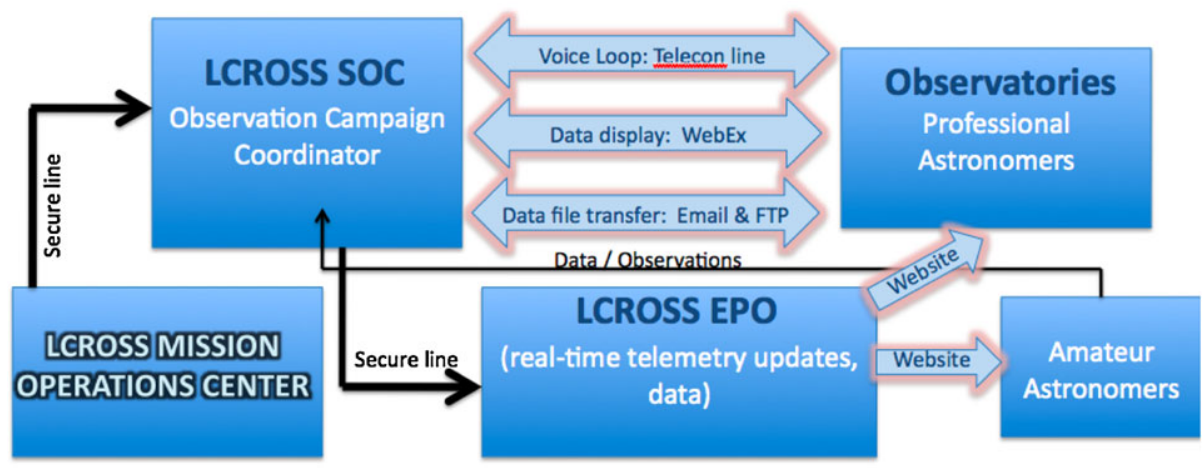

Fig. 8 Schematic diagram showing the methods of communication amongst the LCROSS Science Operations Center (SOC), Mission Operations Center (MOC), Education and Public Outreach (EPO), plus professional and amateur astronomers

night of impact. Multiple mission simulations were conducted to test communications systems in the months prior to impact. A full Operational Readiness Test (ORT) was conducted by the OCC and the distributed Astronomer teams the night prior to impact as a final test of all systems and communications between the LCROSS Project and the observatories.

The LCROSS Observation Campaign Coordinator (OCC) served as the prime source of information from the LCROSS Project to the Astronomers. A schematic depicting the communications flow is shown in Fig. 8. The OCC was physically situated in the Science Operations Center (SOC) at NASA Ames Research Center during the mission. The LCROSS spacecraft was controlled by the LCROSS Mission Operations Center (MOC) at NASA Ames. A secure voice loop and telemetry line between the SOC and MOC provided mission updates from the MOC to the OCC in the SOC. The OCC then filtered this information to extract only the data relevant to the Astronomers' needs to enable their observations. In addition to these direct communications with the professional astronomer teams, the OCC also relayed mission information relevant to observing the impacts to the LCROSS Education and Public Outreach (EPO) team. The EPO team then updated the public LCROSS website with this information which was then available to anyone with internet access, including both professional and amateur Astronomers.

Mission information was relayed from the OCC to the Astronomers via several methods. A voice loop teleconference line was established between the SOC and the Observatories for two-way communications. A WebEx session with secure login was also used such that the OCC could display information and provide updates to the Astronomers via the internet. In addition, email and FTP file transfers were used for a two-way exchange of data and information.

Specific information was relayed from the OCC to the Astronomers at specific times. Communications began at $T-10$ hours to impact (e.g., 10 hours prior to Centaur impact). At this time, the OCC provided a status and position update on the Centaur-Shepherding Spacecraft stack, the expected time of separation for the Centaur and Shepherding Spacecraft, and the expected impact location and time based on the most current trajectory information. At $T-9$ hours 40 minutes the OCC provided a confirmation of the successful Centaur separation with an update on the status of the Spacecraft and expected trajectory. From $T-8$ hours to $T-1$ hour, hourly updates were provided regarding the Centaur and Spacecraft position and status as well as the continually refined impact time and location predictions. At $T-1$ hour the OCC conducted a poll of Astronomers to confirm nominal 
communications between the SOC and each observatory, and also to ascertain information on observatory conditions (including instrument and telescope operations as well as weather status). From $T-50$ minutes through $T-10$ minutes, the OCC provided updates every 10 minutes with refined impact time and location predictions as well as Spacecraft payload operations (the LCROSS payload was activated at $T-50$ minutes). These updates increased in frequency to once per minute from $T-9$ minutes to $T-1$ minute. During the final minute before impact, continuous updates were provided from the SOC based on the real-time mission data from the MOC. Confirmation of impact was provided at $T-0$ and real-time updates provided from $T=0$ to $T+4$ minutes based on the real-time viewing in the SOC of the Spacecraft data collected and returned to Earth during the Flash, Curtain, and Crater phases of the impact. At $T+\sim 4$ minutes the SOC provided confirmation of Shepherding Spacecraft impact.

\section{LCROSS Impact Observations}

Numerous professional observatories and orbiting spacecraft participated in the LCROSS Observation Campaign (Table 2). Given the different telescopes and available instrumentation for each observing asset, specific observing plans and techniques were generated for each observatory. Pre-impact test runs as well as data collection on the actual night of impact were typically conducted. The following sections detail the observing strategies and implementation for several of the participating observatories.

\subsection{Air Force Advanced Electro Optical Systems (AEOS) Telescope, Hawaii (Infrared observations)}

\subsubsection{Planned Observations and Observing Techniques}

The AEOS Infrared group employed a 3-14 micron spectrograph, 3-5 micron camera, and a visible CCD imager operating at 0.7 microns to obtain observations of the LCROSS impact and the impact site on the Moon using the AEOS 3.6 meter telescope from the Maui Space

Table 2 List of participating observatories in the LCROSS Observation Campaign and telescope locations

\begin{tabular}{ll}
\hline Observatory & Location \\
\hline AEOS & Haleakala, Hawaii \\
Allen Telescope Array & Hat Greek, California \\
Apache Point Observatory & Sunspot, New Mexico \\
Earth Observing-1 satellite & Earth orbit \\
Gemini North Telescope & Mauna Kea, Hawaii \\
Hubble Space Telescope & Earth orbit \\
Keck Observatory & Mauna Kea, Hawaii \\
Magdalena Ridge Observatory & Socorro, New Mexico \\
MMT Observatory & Tucson, Arizona \\
Mount Lemmon and Bohyunsan & Tucson, Arizona and \\
Observatories & Youngcheon, South Korea \\
Mount Wilson & Mount Wilson, California \\
NASA IRTF & Mauna Kea, Hawaii \\
\hline
\end{tabular}


Surveillance Site. The primary goals of the observations were flash detection and measurements of the amount and nature of the dust and any water vapor excavated by impact. All instruments performed nominally and data were collected during the impact time frame and immediately afterwards.

The suite of instruments employed to observe the LCROSS impact and impact site are used normally for observations of stars, satellites, and resident space debris. The instruments are positioned part way down the coude optical train (after the primary, secondary, tertiary, and one folding flat). At this point, the beam is intercepted by an off-axis parabola that, together with a small hyperboloid, reimages the beam and converts it from $f / 200$ to f/32. A beamsplitter transmits the light below 1 micron and passes light to the visible CCD imager. The beamsplitter reflects the longer wavelength infrared light towards the window of the spectrograph. The window, which is mounted at 45 degrees to the optical axis, has an anti-reflection coating designed to reflect a portion of the 3-5 micron light to the MWIR camera. The latter is a model LumaDR from Santa Barbara Focal Plane. Its 3-5 micron filter has a notch from 4.2-4.6 microns to reject much of the light in the $\mathrm{CO}_{2}$ band where the atmosphere is opaque. The image scale of the camera is 0.33 arcseconds/pixel and the unvignetted field of view is about 1 arcminute. Most of the 3-5 micron light and almost all of the light from 5 to 14 microns passes into the spectrograph. The spectrograph is the Broadband Array Spectrograph System (BASS) developed at The Aerospace Corporation. It is composed of two channels, each with a double-pass prism and 58 element linear Si:As Back-Illuminated Blocked Impurity Band (BIBiB) array The prism for the MWIR array (2.9-6.7 microns) is $\mathrm{CaF}_{2}$, while the LWIR (6.2-13.9 microns) prism is $\mathrm{NaCl}$. BASS is a low resolution instrument ( $\lambda / \delta \lambda=30$ to 120 , depending on wavelength) optimized for precision spectrophotometry by imaging the pupil on the detector arrays (Hackwell et al. 1990; Russell et al. 2005; Skinner et al. 2007; Skinner et al. 2009).

AEOS science objectives for the LCROSS observations were set largely by the instrumental capability. Like all of the observing teams, we would have liked to detect water ice or water vapor bands (both of which would be seen in a few channels), but low resolution spectroscopy is not suited to the detection of single molecular lines. Rather the BASS instrument provides very wide wavelength coverage extending into the telluric absorption bands and is thus better suited to measuring continuous (e.g., thermal) emission processes. To our knowledge, BASS was the only spectrograph operating in the thermal infrared (beyond 5 microns) to observe the LCROSS impact, and its focus was to help characterize the size, number (or mass) and nature of the solid particles excavated. Since the bulk of the material raised by the impact was non-volatile solids, the size of the particles and their number is essential in determining the mass fraction of any water that might be present in either form.

\subsubsection{Pre-Impact Planning and Test Runs}

The preparation for observing the LCROSS began in September during a similar lunar phase to the night of 9 October 2009 (LCROSS impact night) and included an additional test run on the night of 8 October 2009. A significant amount of the preparatory time was spent unambiguously identifying locations on the south pole of the Moon, and practicing reacquiring these locations and positioning the beam on the impact site. By October 7, images with location markers provided by the LCROSS team had been matched with images from our MWIR camera and by the end of October 8 the impact site could be located rapidly. This was aided by the fact that MWIR images appeared very similar to visible images since the sunlit regions of the Moon, bright in reflected light, are hotter and thus brighter in the infrared emission seen in the MWIR camera images. Observational sensitivity for these 
measurements was not limited in the usual sense by photon counts but rather by detecting the very small changes produced by the impact against the very bright background of the Moon. Accordingly, our observing plan was to begin collecting data shortly before impact and continue in exactly the same mode during the instant of impact and through plume development. The "before" images and spectra would then be subtracted from the "during" and "after" images to look for the small differences due to impact.

\subsubsection{Impact Night Execution}

On the night of 9 October 2009, the weather was clear and seeing conditions were good. Calibrations began approximately 1.5 hours before impact which involved observations of infrared standard stars (stars with known spectral energy distributions) for the flux calibration of the lunar images and spectra. Routine observations of an extended black body were also collected as part of the set-up and calibration for the AEOS telescope. In this manner both the point source extinction data and the extended source responsivity data can be used in the data reduction and analysis processes. The probable impact site on the Moon was located approximately 45 minutes before the impact time and the team acquired a series of practice observations to ensure the instruments and data acquisition system were functioning properly. The relative location of the BASS spectrograph field on the MWIR lunar images was measured on many nights and zenith angles and confirmed during the calibration data collects immediately prior to the impact time. Because the MWIR camera observes over much of the same wavelength region covered by the spectrograph, there is no differential refraction between the fields-of-view of the two instruments.

\subsubsection{Summary of Techniques}

Actual impact observations began with the BASS spectrograph and the MWIR camera 60 seconds prior to impact. The BASS instrument acquired spectra 200 times per second with the MWIR camera framing 100 times per second. Data collection continued in this manner until after the impact of the Shepherding Spacecraft.

\subsection{Allen Telescope Array, California (Radio Observations)}

\subsubsection{Planned Observations and Observing Techniques}

We planned to observe Cabeus Crater at the lunar south pole during the LCROSS impact on 9 October 2009 with the 42-element Allen Telescope Array (ATA) at Hat Creek Radio Observatory. There were two goals for the observations: 1) to detect the presence of $\mathrm{OH}$ in the impact plume as evidence for water on the surface, and 2) to detect the impact in the continuum to better understand impact flashes caused by meteor strikes on the lunar surface typically seen only at optical wavelengths. The ATA observed the crater at three frequencies: 1666.5 MHz, 1720.5 MHz and 7000 MHz. We chose 1666.5 MHz to observe two OH mainline $18 \mathrm{~cm}$ transitions in one band, the $1665.4 \mathrm{MHz}$ line and the $1667.3 \mathrm{MHz}$ line, and we observed at $1720.5 \mathrm{MHz}$ to search for a third $\mathrm{OH}$ spectral line. We observed the impact in the continuum at $7000 \mathrm{MHz}$ as well. Cabeus Crater was observed from 09:32:45 UTC until 14:57:55 UTC on 9 October 2009.

Both dual-polarization correlators were employed, one tuned to $1666.5 \mathrm{MHz}$ with a bandwidth of $6.55 \mathrm{MHz}$ and the other to $7000 \mathrm{MHz}$ with a bandwidth of $104.86 \mathrm{MHz}$. A total of $25 X$-polarization and $24 Y$-polarization inputs were used for this observation that represented 24 dual polarization antennas and one $X$-polarization only antenna. Three calibrators 
Fig. 9 Allen Telescope Array (ATA) data tracking the Cabeus Crater. The crater is centered at $(0,0)$ in the map

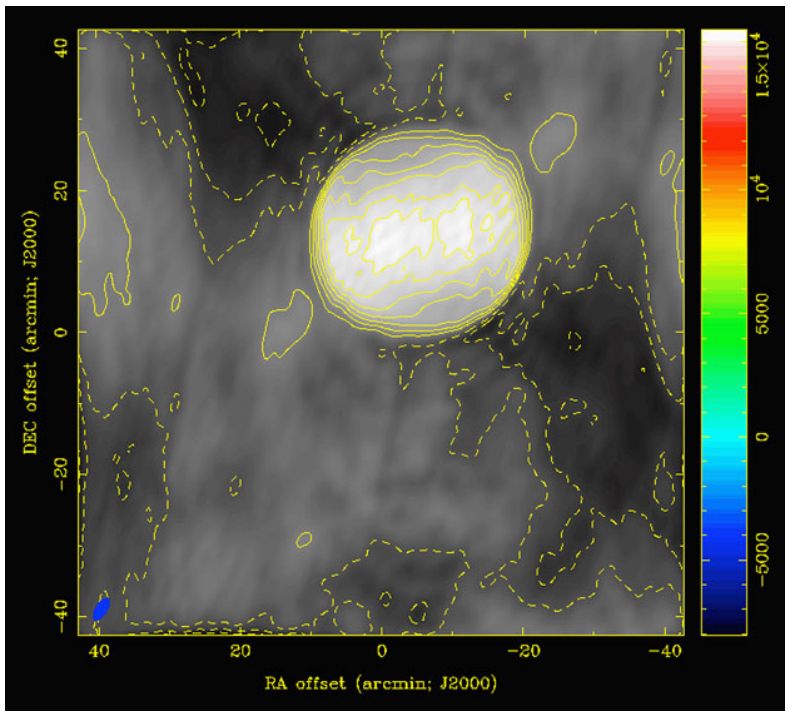

were used, $3 \mathrm{c} 123,3 \mathrm{c} 84$, and 3c147, and all were observed before and after the impact. Correlator dump time was set to 10 seconds. While this time was not optimum for this kind of experiment (a much shorter time would have been preferable), it was necessary to keep both correlators and the beamformer running simultaneously without incurring data capture errors. Starting at 11:00:00 UTC, the crater was observed continuously with 40 minute scans so that at the actual time of impact, 11:31:19 UTC, the telescope would be taking data.

One of the three beamformer back-ends was used to observe Cabeus Crater before, during and after the impact. A total of $23 \mathrm{X}$-polarization and $22 \mathrm{Y}$ polarization inputs were used in this observation, and represented 22 dual-polarization antennas and one antenna for which the $X$-polarization only was preferred. The beamformer was calibrated using an internal correlator and Cassiopeia-A as the strong-source (delay calibrator) and 3c48 as the weaksource (unresolved phase calibrator). Data were recorded for 3.5 hours from 11:04:04 UTC to 14:24:34 UTC (27 minutes before impact and 3 hours afterwards). This recording yielded approximately $144 \mathrm{~GB}$ of raw data.

An interesting issue that had to be addressed involved the tracking of the crater at the lunar south pole. Ephemeris files generated by the ATA software system track the centroid of the Moon. In order to achieve the best sensitivity, the crater should be positioned in the center of the beam, so an offset ephemeris had to be created. An offset ephemeris file was generated using a program that modified data from the Jet Propulsion Laboratory (JPL) Horizons ephemeris generator. In order to use the new ephemeris file for the ATA, another program modified the crater ephemeris file so that the ATA software could read the coordinates. Figure 9 shows a map of the Moon at $1720.5 \mathrm{MHz}$ where the impact crater Cabeus is in the center of the primary beam.

A second consideration was determining the frequency to best observe the continuum. We wanted to observe a frequency at the upper range of the band and so had to image the Moon at $500 \mathrm{MHz}$ intervals between $6 \mathrm{GHz}$ and $10 \mathrm{GHz}$ to ascertain where we could create a good image. We chose $7 \mathrm{GHz}$, as above that frequency, image quality deteriorated due to the small beam size at higher frequencies and the large size of the Moon. 


\subsubsection{Pre-Impact Planning and Test Runs}

The first planning activity was submitting proposals to the ATA scheduling committee to get time for testing tracking, observing during the group testing sessions, and sufficient time before and after the actual impact. This task was accomplished readily as it was such a unique event and we were the only $\mathrm{cm}$ wave radio interferometer in the LCROSS Observation Campaign. The first tests involved tracking the crater and were performed over several hours at 1665.5 MHz and 1720.5 MHz using the offset ephemeris file (see Fig. 9). Tracking tests were verified during subsequent group testing/rehearsal sessions.

\subsubsection{Impact Night Execution}

Observations were set up several hours prior to impact and we observed the $1665.5 \mathrm{MHz}$ and 1667.4 MHz line of $\mathrm{OH}$ in $\mathrm{W} 3(\mathrm{OH})$ to verify that we were detecting $\mathrm{OH}$ with the instrument. We observed with the correlators two hours prior to impact and 3.5 hours post-impact. The beamformer was set up an hour prior to impact and we had all backends running at the time of impact, 11:31:19 UTC. The beamformer also continued observing for three hours postimpact. Data accumulation went well and no problems were encountered collecting the data required to perform our analysis.

\subsubsection{Summary of Techniques}

The observing plan worked well and no issues were encountered during the time of the impact. We utilized the full capability of the ATA and collected as much data at as many frequencies as the instrument would allow. Overall, we collected data at $1666.5 \mathrm{MHz}$, $1720.5 \mathrm{MHz}$ and $7000 \mathrm{MHz}$, representing a wide range of frequencies at which to observe the impact.

\section{Apache Point Observatory, New Mexico (Visible-Infrared Observations)}

\subsection{Planned Observations and Observing Techniques}

The New Mexico State University-NASA/Marshall Space Flight Center (NMSU-NASA/ MSFC) team planned to conduct high time series imaging of the impact plume with the goal of characterizing the lunar regolith within a permanently shadowed crater near the south pole of the Moon. We used a multi-telescope approach to image the LCROSS plume, making use of three different facilities with video or high-speed imaging capabilities. The spectral coverage afforded by the combination of the three instruments used on these three telescopes spanned $0.47-1.9$ microns.

We planned to observe the LCROSS impact with two telescopes at the Apache Point Observatory (APO), which is located in the Sacramento Mountains. We also employed the Tortugas Mountain Observatory (TMO) in Las Cruces, NM. We used the Agile camera on the Astrophysical Research Consortium (ARC) 3.5-meter telescope at APO to acquire images of the LCROSS impact region. Agile is a high-speed time-series photometer based on the Argos camera at McDonald Observatory (Nather and Mukadam 2004). We employed a StellaCam EX (Sony HAD EX chip) video camera on the NMSU 1-meter telescope at APO through a Johnson-Cousins R filter. The $720 \times 480$ pixel 30 frame per second 8-bit data were recorded to a PC. The NIR camera used at Tortugas Mountain Observatory (TMO) is 
Fig. 10 Data from Apache Point Observatory. The Agile dark slide was used as a mask to block out a portion of the Moon's illuminated disk within the Agile field of view. The dark region with the rounded edge on the right clearly shows the dark mask. Analysis of a dome flat image taken with the dark slide inserted to the same point showed that because the dark slide is slightly defocused, vignetting effects result in image attenuation out to the region of the vertical dashed line, as depicted in the lower panel. We optimized our placement of the LCROSS target crater, Cabeus (circled), to be outside of the dark slide attenuation limit

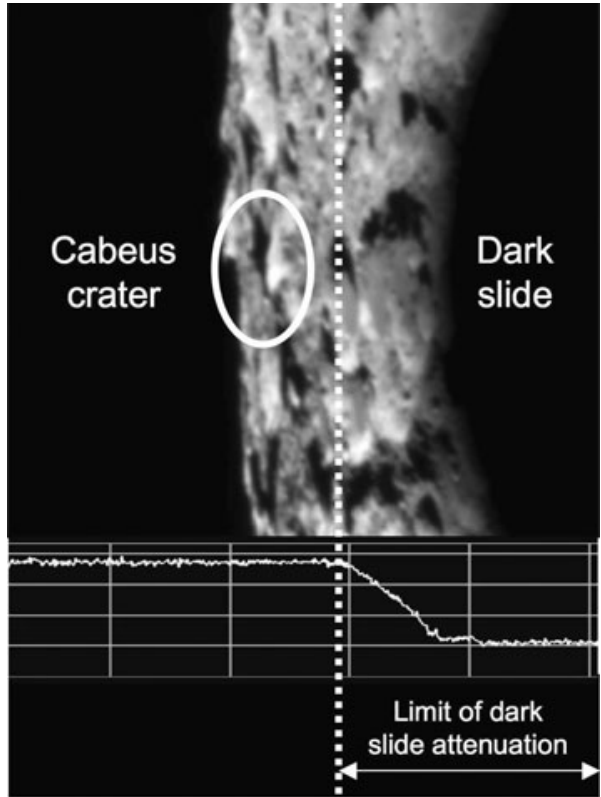

a Goodrich Sensors Unlimited SU640KTSX, similar to those used on the LCROSS Shepherding Spacecraft (Colaprete et al. 2010). The $640 \times 512$ pixel 30 frames per second 12-bit data were recorded to a Boulder Imaging Quazar video recording system.

For the observations with the ARC 3.5-m telescope, we implemented a makeshift "lunagraph" to block out most of the illuminated disk of the Moon. We inserted the Agile dark slide partway into its slot, thereby creating a dark mask with a curved edge. We used a dome flat image to identify the pixel limits of the attenuation due to the dark mask. A sample image of the Moon taken with the mask in place is shown in Fig. 10. This proved to be a simple and effective way to reduce scattered light from the Moon during our attempt to image the faint plume.

\subsection{Pre-Impact Planning and Test Runs}

Because our science team was geographically distributed between New Mexico and Alabama, we conducted monthly planning teleconferences for roughly a year leading up to the impact event. In parallel with these teleconferences, we conducted several test runs with each telescope to test the following aspects of the observations:

Navigation Our most important objective was to become familiar enough with the Moon's topography near the south pole that we could quickly and accurately ensure that we were observing the correct location on the night of impact. A primary goal of our test observations was thus to obtain images of the LCROSS target region with illumination conditions as close as possible to those expected at impact. This included the same (or similar) lunar libration, and the same Sun-Earth-Moon illumination angle, or lunar phase. Our test observations revealed that even small variations in illumination angle (i.e., just one day from ideal) resulted in very different shadow conditions near the lunar limb and terminator. As a product of our practice imaging, we generated a widely distributed map of the Moon's South Polar region with illumination close to that expected on the night of impact, clearly 


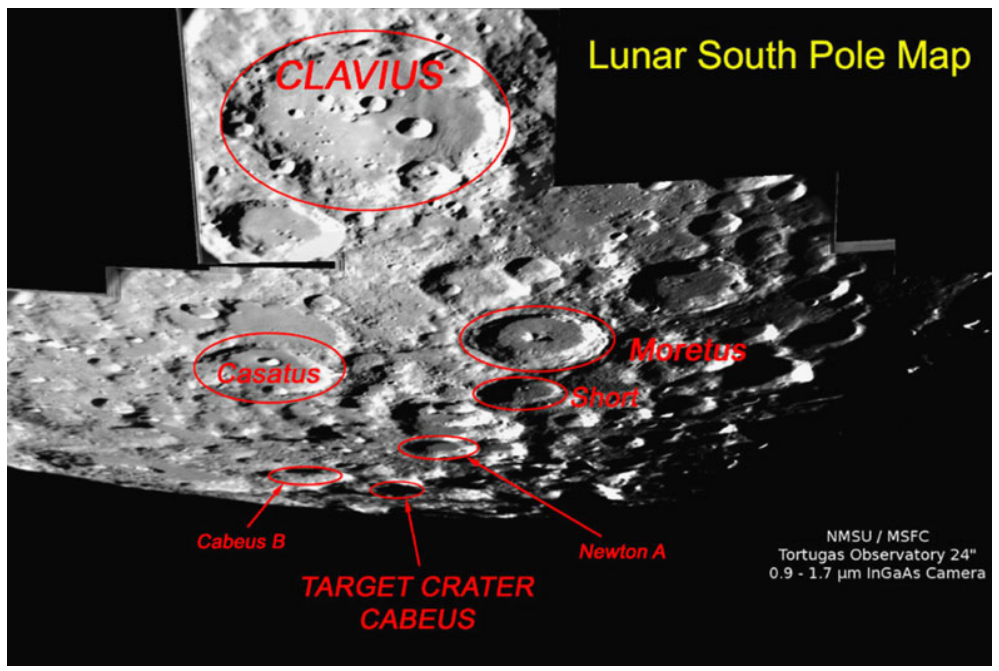

Fig. 11 Image of LCROSS target crater generated from near-IR video taken at the Tortugas Mountain Observatory 0.6-m telescope in Las Cruces, NM. The image was taken on August 11, 2009, during a practice observing session and was shared as a planning tool with all ground-based observing teams. There is some distortion toward the edges of the map resulting from the automated image combining method used to generate the map

indicating the Cabeus Crater (Fig. 11). Our maps were also used by NASA to calibrate their digital elevation models and shadow maps that were used as planning tools for the LCROSS impact observations.

Pointing and Tracking The ARC 3.5-m telescope is used for APOLLO, the APO Lunar Laser-ranging Operation (Murphy et al. 2008), so it routinely tracks specific locations on the Moon. We specified our target locations in selenographic coordinates and slewed to a recognizable crater near the LCROSS target area using additional tracking velocities so that the telescope would track at the lunar (non-sidereal) rate, then used offsets to optimize the pointing. We found that the tracking rates were valid for about half an hour, so we slewed with a rate calculated for 15 minutes in the future, then reslewed again when that rate was 15 minutes old. We followed a similar strategy for the NMSU 1-m telescope; we issued offset tracking velocities once we pointed the telescope at the target crater. The 0.6-m TMO telescope was adjusted manually by issuing minor adjustments to the pointing.

Signal-to-Noise and Dynamic Range For the observations planned with Agile on the ARC 3.5-m, we experimented with the combination of several different bandpass filters (MSSS $\mathrm{V}, \mathrm{R}$, and I, and SDSS-z), neutral density filters, and camera readout rates to optimize the instrument throughput and signal to noise ratio. We also wanted to minimize scattered light in our field of view and maximize the dynamic range, which would increase the likelihood of detecting a faint plume against the bright limb of the Moon. As a result of our tests, we decided to use two filter combinations: an ND4 neutral density filter for finding our target location on the Moon, and an MSSS V + ND2.5 combination for observing the impact event. We also had the SDSS-z + ND1.3 combination ready as a backup in case the Moon was obscured by thin clouds on the night of impact. We chose the filter combination that allowed near saturation of the brightest lunar terrain near Cabeus, which was the ridge facing Earth. 
This allowed imaging with the greatest possible dynamic range based on sky conditions at APO the hour before impact. Our plan allowed flexibility to adapt to the specific sky transparency we faced on the night of impact, which could not be duplicated exactly during our test observations. For the two video cameras, combinations of gain and electronic shutter speed were selected so that the brightness of the illuminated disk of the Moon was at the level of roughly $50 \%$ of the dynamic range.

Communications We tested various forms of communication between the three observing stations for our teams. We found that audio signal was essential, while video was not. As the video also required greater internet bandwidth, we opted for audio-only communications on the night of the LCROSS impact.

Data Processing In order to be able to provide quick-look images to the LCROSS team, we developed several automated data reduction routines. These reduced the video data into small (10-second) segments and annotated the CCD images with proper identification.

All of our test observing runs were valuable and provided critical information that we used to prepare for the night of impact. Aside from preparing for optimizing our science observations, another extremely useful exercise was our participation in the impact night simulation that was conducted by the NASA LCROSS Science Operations Center (SOC) on UT 15 September 2009. This experience provided the observing teams with a sense of the kind of information that would be transmitted from the SOC to the observers (and at what rate), the kind of information that the SOC expected to receive from the observers, the limitations of data transfer to the SOC for press releases, and the general pace and schedule of events on the night of impact.

\subsection{Impact Night Execution}

The night of impact was carefully scripted, with each of our three observatories following a predetermined timeline. We were all connected via teleconference using the Skype software, which enabled us to ensure real time communication among team members and verify that all three observing teams were on schedule. We also had one team member in the APO $3.5-\mathrm{m}$ control room who served as our Communications Officer; he was the single point of contact for the NMSU-NASA/MSFC team to the LCROSS SOC. He provided status reports to the LCROSS SOC when queried, and disseminated Spacecraft information as it was reported to the observing teams by the SOC.

With the 3.5-m telescope we imaged the Moon continuously during the time span 11:19:26-12:04:26 UT in an effort to completely characterize the Cabeus Crater region before, during, and after the LCROSS impact, which occurred at 11:31:19 UT. The data set is archived in NASA's Planetary Data System (PDS) and is available for download by the general public (Chanover 2010). The $1-\mathrm{m}$ and 0.6 -m video recordings were started approximately 10 minutes before impact and ran for 50 minutes post impact.

\subsection{Summary of Technique}

Our plan for imaging the impact plume was executed flawlessly. Each team collected the volume and quality of data expected from the ARC 3.5-m, the NMSU 1-m, and the TMO 0.6-m telescopes. The most valuable lesson learned in this campaign was the importance of involving ground-based observers early on in the planning stages for an event such as the LCROSS impact. Because the ground-based observers were stakeholders even in the mission planning stages, we were able to provide and receive input regarding the LCROSS impact location and its observability from Earth. 


\section{Bohyunsan Observatory (South Korea, Visible Observations)}

\subsection{Planned Observations and Observing Techniques}

Planned observations at BOAO (Bohyunsan Optical Astronomy Observatory) focused on spectroscopy. We planned to observe the expanded $\mathrm{OH}$ exosphere four hours after the impact. The timing of these observations was driven by the local moonrise in Korea. We utilized the BOAO $1.8 \mathrm{~m}$ telescope equipped with a long-slit spectrograph. The slit length was set to $3.6 \operatorname{arcmin}(15 \mathrm{~mm})$ which is equivalent to $160 \mathrm{~km}$ on the lunar surface, and the slit width was set to 2 arcsec during the observing run. A Tek $1 \mathrm{~K}$ CCD camera (24 microns/pixel) was employed for the long-slit spectrograph. The 1200 lines/mm grating was applied for the resolution of 0.7 angstroms/pixel. The wavelength covered the range of 565$634 \mathrm{~nm}$ and the central wavelength was set to $600 \mathrm{~nm}$ for the best detection of $\mathrm{H} 2 \mathrm{O}+$ line $(\lambda=619 \mathrm{~nm})$. A Fe-Ne lamp was used for the wavelength calibration. We obtained $\mathrm{Fe}-\mathrm{Ne}$ comparison spectra before and after each object exposure for accurate calibration of the wavelength. We also obtained quartz lamp exposures for flat fielding, twilight exposures for illumination corrections, and standard star (HR 718) frames during the observing run.

\subsection{Pre-Impact Planning and Test Runs}

We performed telescope pointing tests for BOAO a day before the impact. However, precise pointing was not necessary for BOAO since we could not locate the exact position along a largely expanding $\mathrm{OH}$ cloud. Thus, we roughly targeted the expected impact site on the Moon and performed test observations. We also checked the pointing and tracking systems of the BOAO $1.8 \mathrm{~m}$ telescope during this observing run.

\subsection{Impact Night Execution}

At BOAO, the observation was started on 15:10 UT and continued until sunrise at 19:53 UT. The spectroscopic observations of the LCROSS impact were made ON- and OFF-exposures. For ON-exposures, the slit was positioned to the perpendicular direction against the edge of the horizon of the Moon, and for OFF exposures, the slit moved along the slit to the outside of the Moon. The center of the slit was carefully placed on the impact site. The ONexposures were contained with the Moon on the half of the slit, and sky and scattered lights on the other half. The exposure time was 0.4 seconds for ON-, and 25 seconds for OFFexposures. We also acquired a total of $42 \mathrm{ON}$ - and $39 \mathrm{OFF}$-spectra. A standard star (HR 718) was observed between the ON- and OFF-spectra observations with twilight frames at the end of the whole observation process. We obtained a total of 17 spectra of the standard star with several sky and comparison spectra for this study.

\subsection{Summary of Techniques}

Although several $\mathrm{OH}+$ and $\mathrm{H} 2 \mathrm{O}+$ emission lines were detected, it is difficult to discriminate those signatures from the terrestrial atmospheric origins.

\section{Earth-Observing-1 (EO-1) Observations, Earth Orbit (Visible-Infrared Observations)}

\subsection{Planned Observations and Observing Techniques}

The Earth-Observing-1 (EO-1) satellite is an Earth-observing spacecraft flying in a sunsynchronous orbit about the Earth at a current mean altitude of $695 \mathrm{~km}$. The EO-1 mission 
planned to observe the LCROSS lunar impact using its two imaging instruments. The first of these is the Advanced Land Imager (ALI), a 9 band multi-spectral instrument covering the electromagnetic spectrum from 0.4 to 2.5 micrometers in a 4 sensor chip array configuration. Eight of the ALI multispectral bands have 320 cross-track pixels, each with an instantaneous field of view (ifov) of 8.9". The panchromatic band has 3 times better resolution with 960 cross-track pixel and a pixel ifov of 2.9". At the mean lunar distance for the Earth, these translate to pixel resolutions of $16.1 \mathrm{~km}$ and $5.4 \mathrm{~km}$. The second imaging instrument on EO-1 is the Hyperion. This is an imaging spectrometer covering the same wavelength range as the ALI but with 196 bands, each with 10 nanometer bandwidth. The Hyperion uses 256 cross-track pixels with an ifov of $8.9^{\prime \prime}$.

The primary goal of the EO-1 observation of the LCROSS lunar impact was to examine the spectral data provided by the Hyperion for water content.

\subsection{Pre-Impact Planning and Test Runs}

Early in EO-1 planning, the strategy was to time the already-existing lunar calibration command sequence to image the Moon using Hyperion during the time of the lunar impact. However, the planned lunar impact time (11:31:19z) dictated that the lunar calibration sequence occur during EO-1's orbit day. This imposed a severe impact on spacecraft power. Due to this power concern, the EO-1 imaging plan was modified to include imaging the Moon during the first orbit night after the LCROSS impact. To establish a pre-impact baseline, lunar images would also be acquired two EO-1 orbits prior to the post-impact image (Fig. 3a). Allowing for a full spacecraft orbit to transpire between the pre- and post-impact calibrations would allow the battery to reach a full state of charge between the two imaging events. This modified plan was used for creating the command load for EO-1's support of the LCROSS mission.

\subsection{Impact Night Execution}

A stored absolute time sequence command load was used to command the spacecraft during the day of the LCROSS impact. Hence, the spacecraft could perform these activities even when not in contact with the EO-1 Mission Operations Center. The pre-impact lunar calibration was scheduled to begin at 09:05z, while the post-impact calibration was scheduled to begin at 12:23z. The pre- and post-impact data were downloaded properly, with impact-day evaluation showing that the images were taken successfully. No significant limit violations were experienced by the spacecraft during the execution of these activities.

\subsection{Summary of Technique}

Preliminary analysis of the ALI and Hyperion data performed in the days after the LCROSS impact showed that the spectral data were successfully captured by the pre- and post-impact images.

\section{Gemini North Telescope, Hawaii (Infrared Observations)}

\subsection{Planned Observations and Observing Techniques}

The objective of the Gemini-North LCROSS remote sensing observations (Program ID GN-2009B-Q-35) was to observe the temporal and spatial evolution of the impact plume 
Fig. 12 Model spectral shape (slope) for grains with sizes 1 , 10, 30, 100 microns derived from surrogate pyroxene grains to replicate the lunar regolith with the NIFS spectral bandpass indicated by the vertical solid lines

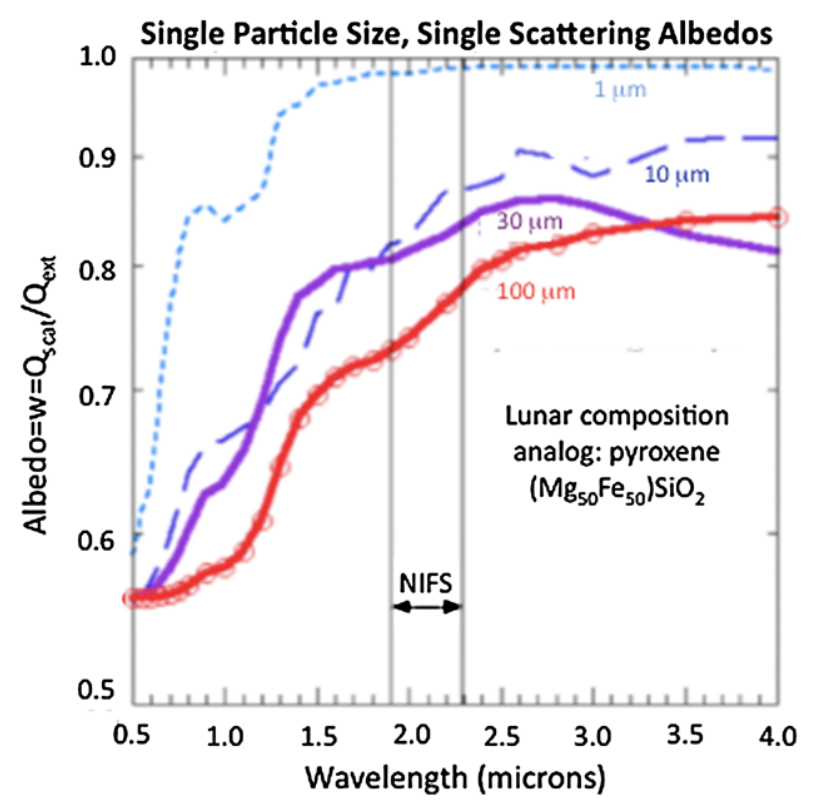

using Near-Infrared Integral Field Spectrograph (NIFS; McGregor et al. 2002) at wavelengths from 1.9 to 2.3 microns (spectral resolving power $R=\lambda / \Delta \lambda \approx 5000$ ) using the $\mathrm{K}$ band grating in seeing limited mode (typically 0.4 arcsecond full-width-at-half-maximum or larger). Although the ejecta curtain evolved rapidly, our experiment was designed to utilize the Integral Field Unit's 3 arcsecond $\times 3$ arcsecond field-of-view (equivalent to $6 \mathrm{~km} \times$ $6 \mathrm{~km}$ equivalent projected distance on the Moon), and select integration cadence observe the dense core of the ejecta curtain in the first 30 seconds after impact, and measure the spreading plume over 8 minutes as the ejecta overfills the spectrograph's field-of-view. The twodimensional spatial coverage (containing $29 \times 58$ image slices or slits over the focal plane) of NIFS has powerful advantages over remote sensing observations of the LCROSS event observed by single-slit spectrometers. NIFS can see the entire plume, whereas a single-slit instrument can only see a part of the plume at once. Furthermore, NIFS spectral data cube from 1.9 to 2.3 microns uniquely enables the de-convolution of the particle size distribution (Fig. 12) and the surface density distribution from the observed surface brightness, providing a critical assessment of the ejected mass and hence the ice concentration.

\subsection{Pre-Impact Planning and Test Runs}

In order to verify that we could point the Gemini telescope with sub-arcsecond precision and lock guiding loops, four separate on-sky engineering runs were scheduled prior to the LCROSS impact event. These runs were selected based on when the Moon's lunation and libration would most closely match the illumination conditions at the impact site on the actual event date. The first challenge was to track a specific location on the Moon, which is not supported by the non-sidereal target query capability in the Gemini Observing Tool. The lunar track rates are too high and changing too quickly to be input manually as was immediately obvious during the first engineering trials. Fortunately a solution was found whereby detailed ephemeris files could be read directly into the Gemini telescope control system (TCS). 
The second challenge was to establish guiding, since the Gemini telescopes are lightweight and susceptible to windshake and all observations must be guided in order to ensure the best image quality. Unfortunately we could not guide on a nearby star, because the lunar track rates are so high that any background guide star would move completely through the patrol field of the peripheral wavefront sensors during the one hour duration of the planned LCROSS observations. Attempts to guide on the surface of the Moon with the peripheral wavefront sensor met with mixed success, as the illuminated surface features saturated the guider detectors. We were able to guide for limited periods of time using the bright peaks of lunar surface features rising above the shadows on the dark side of the Moon, but these peaks have limited visibility lifetimes as the terminator marches across the face of the Moon. Furthermore, scene descriptor resources that would have enable observers to predict in advance and to visualize the locations of properly illuminated lunar features did not exist. The "work-around" solution that enables guiding was to use the ALTAIR adaptive optics wavefront sensor in a very limited mode. ALTAIR is equipped with a neutral density (ND) filter providing $\sim 8$ magnitudes attenuation at optical wavelengths (band center near $550 \mathrm{~nm}$ ); deployment of the ND allowed Gemini to guide on the bright side of the Moon without saturating. However, there were no features within the 25 arcsec patrol radius of the impact site which were adequately point-like to allow for full adaptive optics correction. Instead, we discovered that by disabling all of the voltages on the deformable mirror permitted use of only the tip-tilt stage. This novel approach allowed the Gemini North telescope to compensate for windshake and potential tracking errors, delivering stable, seeing limited images to the NIFS science instrument for the duration of the LCROSS observations (Fig. 13).

\subsection{Impact Night Execution}

The integration times $(5 \mathrm{sec})$ and duty cycle of the spectrometer readout $(15 \mathrm{sec})$ and execution overhead $(6 \mathrm{sec})$ were carefully chosen so that a single data-cube would take 26 seconds to complete. NIFS observations commenced approximately 11:30:56.0 UT (approximately $60 \mathrm{sec}$ prior), with the next data cube integration commencing at 11:31:22.5 UT after Centaur impact (occurring at 11:31:19 UT). We continued to observe the Centaur impact site with NIFS and a $26 \mathrm{sec}$ data cube cadence until 11:47 UT, well after the Shepherding Spacecraft secondary impact. This observational strategy enabled us to obtain spectral cubes prior to impact for back-ground subtraction and cirrus compensation calibration, lock the telescope guider loops, establish the best-focus and respond to last minute trajectory updates from the LCROSS Science Operations Center.

The Gemini NIFS data were reduced by carefully following the detailed cookbook provided by the NIFS instrument team (Turner et al. 2006). The complex reduction is performed in three main steps using the reduction package IRAF, and involves: 1) reduction of the baseline calibration data (which includes flats and darks); 2) reduction for telluric standard calibrations; and 3) reduction of the primary science data. The data processing steps went smoothly and we have high confidence that the final NIFS products were reduced and calibrated accurately.

\subsection{Summary of Technique}

The resulting analysis of the NIFS data set presented a few challenges. The primary issue is the background subtraction. Few, if any, of the pre-impact data adequately removed the background from the post-impact data. Seeing and transparency variations (thin cirrus were present over Mauna Kea) were large enough that even after background subtraction, some 


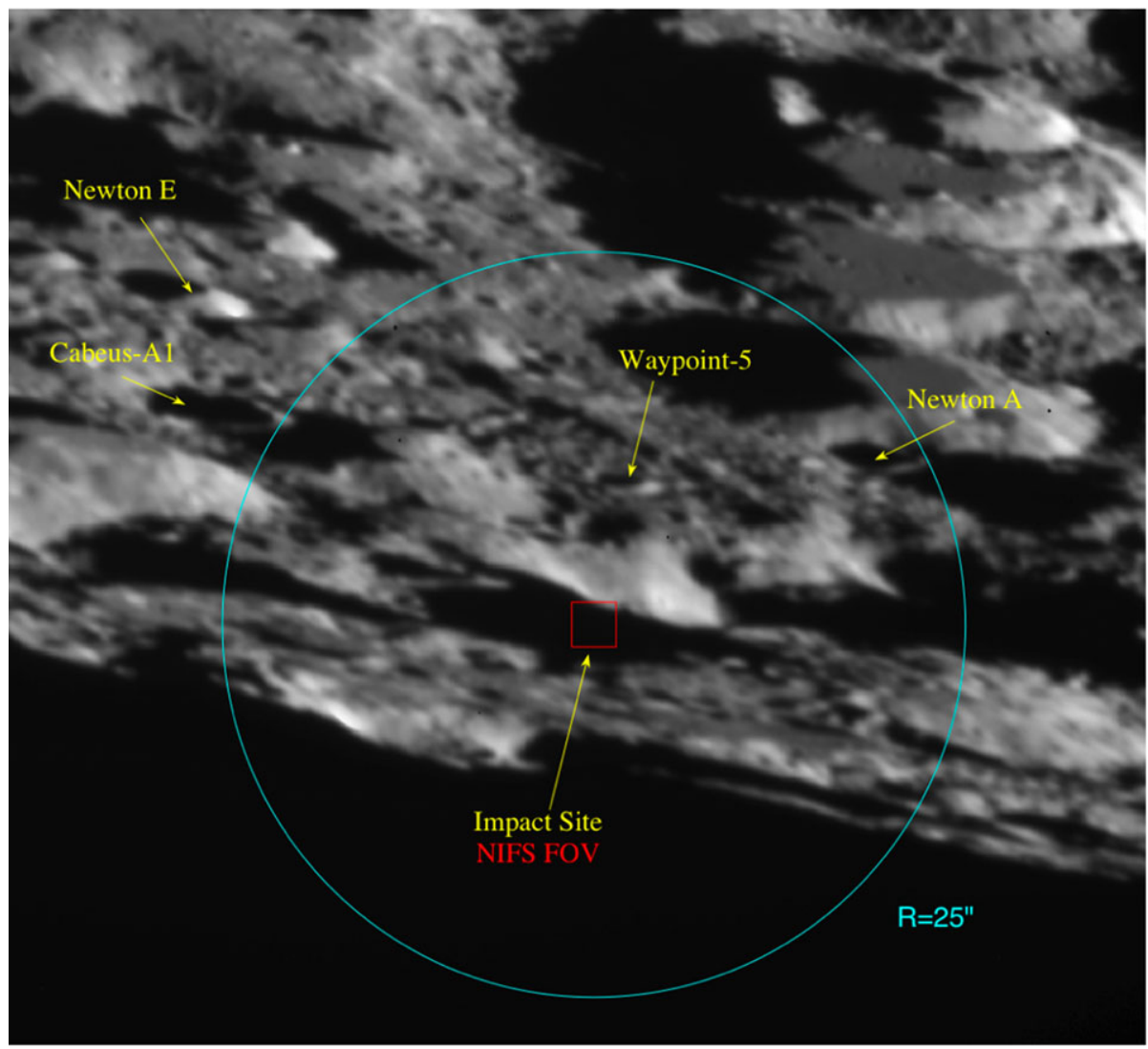

Fig. 13 Gemini-North GMOS image of the 2009 October 09 UT LCROSS impact site near the crater Cabeus near the southern lunar pole. During the night of Centaur impact, the telescope was guiding on a lunar crater designated "Newton-A" with tip/tilt only corrections applied to the system. The NIFS IFU field of view is inset in the small red-box and the radius of the guider patrol field indicated by the blue-green circle

residual light from the terrain was always present at the target location. The residuals provide a systematic error to our observations. With the presence of this systematic error, we have been unable to identify (above the 3-sigma level) any spectral features attributable to water bands from sublimating ices intermixed in the ejecta regolith, or significant changes in the spectral slope over the NIFS bandpass pre- versus post-impact due to dust entrained in a post-desorption (ice-free) impact plume employing rudimentary analysis techniques.

\section{Hubble Space Telescope, Earth Orbit (Ultraviolet-Visible Observations)}

\subsection{Planned Observations and Observing Techniques}

The great strengths of the Hubble Space Telescope (HST) are high resolution imaging and spectrographic sensitivity in the vacuum ultraviolet. Our observations were planned to capitalize on these strengths and to complement the efforts of the other observers. The sunlit lunar disk is too bright to image with HST in all but the narrowest filters and shortest exposure times, and instrument overhead makes observations of the lunar surface an inefficient 
Fig. 14 Schematic showing Hubble Space Telescope pointing for spectroscopic observations. Several instrument fields of view are shown including NICMOS (Near Infrared Camera and Multi Object Spectrometer), FGS (Fine Guidance Sensors), and ACS (Advanced Camera for Surveys) which were not used in this study. STIS (Space Telescope Imaging Spectrograph) and WFC3 (Wide Field Camera 3) were used for LCROSS observations

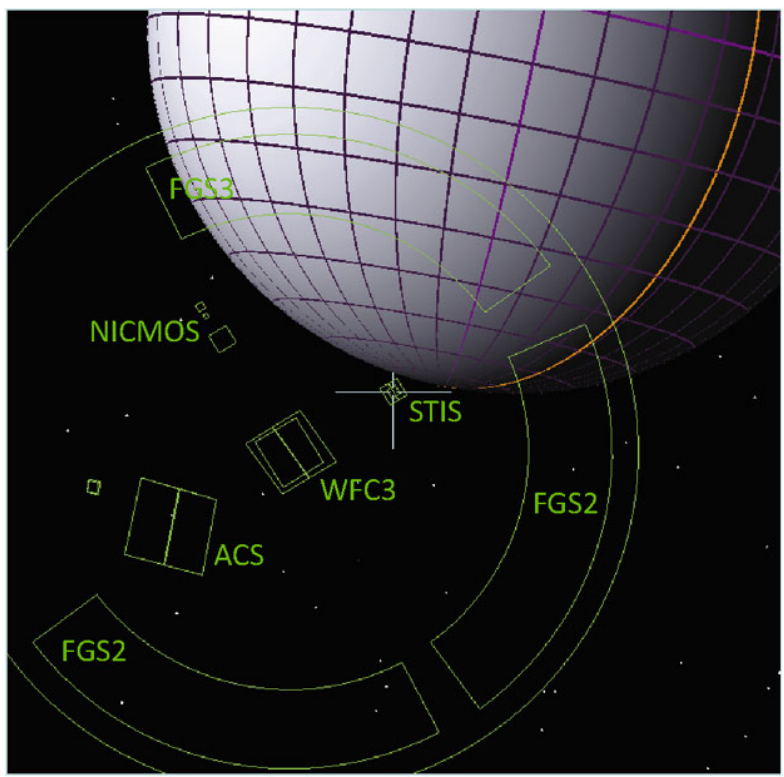

use of spacecraft time. We therefore opted to attempt to image the gas plume rising above the limb of the Moon in the HST orbit that coincided with the LCROSS impact, and to study the composition and evolution of the cloud on subsequent HST orbits using spectral observations in the $210-306 \mathrm{~nm}$ wavelength range. The planned HST instrument pointing is illustrated in Fig. 14.

HST observations are necessarily limited by the spacecraft position in low-Earth orbit. Observations of a given target near the ecliptic plane are typically possible for half of the 96 minute orbit. A further complication arises from parallax produced by HST's orbital motion. For most solar system objects, this apparent motion can be compensated by the telescope's planning and scheduling system. For objects as close as the Moon, however, the ground system cannot correct for the full apparent motion of the Moon as seen by HST. Only when HST is going towards or away from the Moon, or more or less linearly across the Earth as seen from the Moon, can the system reliably compensate for this motion (Roman and Skillman 2007).

Another complication to HST lunar observations is guiding. In normal operation, HST uses Fine Guidance Sensors (FGS) at the edge of its field of view to observe guide stars and maintain pointing to a small fraction of an arcsecond. Close to the Moon, however, the scattered light is too bright for these sensors to work, and indeed for many observations one or more FGS was occulted by the lunar disk. Thus the observations were made under "gyro control". This pointing drifts at a canonical rate of one milliarcsec per second. However, at the time of our observations (immediately following the installation of new gyros, in Servicing Mission 4) the drift rate was about ten times this amount because one of the replacement gyros wasn't well calibrated at the time of our observations (Storrs and Colaprete 2010).

\subsection{Pre-Impact Planning and Test Runs}

We proposed six orbits of HST observations of the LCROSS impact. The objective was to use HST's ability to do high spatial resolution imaging over a large field to provide context 
for the in situ observations of the primary impact plume, and to use HST's ability to do spectroscopy in the vacuum ultraviolet to monitor the temporal evolution of $\mathrm{OH}, \mathrm{CO}, \mathrm{CO}_{2}$, $\mathrm{CS}$, and other radicals as the plume dissipated. The general strategy was to use one HST orbit to observe at the time of the LCROSS impacts and then use a subsequent orbit(s) to observe the $\mathrm{OH}$ - exosphere. The planned orbits and associated observations are described below.

Orbit \#0: STIS CCD G230LB spectroscopy, 0.210-0.306 microns. Pre impact observation to determine the amount of background light in the target vicinity before LCROSS impact.

Orbit \#1: WFC3 F300X imaging. Three pairs of images taken at "good" parts of HST's orbit, to constrain the evolution of the impact plume with high spatial and temporal resolution. These observations were timed such that this orbit occurred simultaneously with the LCROSS impacts. The goal of this spectral range is to observe the rapid 0.3085 micron $\mathrm{OH}$ emission from the water vapor in the hot plasma occurring just after the impact flash. Observation of the first impact position a few minutes prior to impact is desired. Observing the evolution of the second impact flash and the ejecta curtain for at least several hundred seconds is also desirable.

Orbit \#2 (and two subsequent orbits): STIS CCD G230LB spectroscopy, $\sim 0.210$ 0.306 microns. The short wavelength part of the 0.3085 micron $\mathrm{OH}$ band should be observable (for example, this line is quite wide in IUE (International Ultraviolet Explorer) spectra). The $\mathrm{OH}$ exosphere should last for several hours after impact so three HST orbits after the WFC3 observations are ideal. The $\mathrm{OH}$ band at 0.3085 microns is at about as short a wavelength as can be observed from the ground, and as long a wavelength as proposed to be observed here, allowing normalization of the HST results with ground-based results. Monitoring the strength of this band is central to measuring the amount of water evolved in the impact, as water dissociates to $\mathrm{OH}$ and $\mathrm{H}$ very rapidly when exposed to sunlight. The CO Cameron bands (0.190-0.280 microns) derive primarily from the photodissociation of $\mathrm{CO}_{2}$. We proposed to monitor these bands simultaneously with $\mathrm{OH}$ and $\mathrm{CS}$ ( 0.260 microns) to constrain the composition of the volatile fraction of the impact plume.

Orbit \#5 (a day later): A repeat of the spectroscopic observations a day after the impact can constrain long lived radicals and allow a determination of the background emission.

\subsection{Impact Night Execution}

The HST observations were executed exactly as programmed. Data were downloaded through TDRSS (Tracking and Data Relay Satellite System) as planned, arriving at Space Telescope Science Institute starting about four hours after being acquired.

\subsection{Summary of Technique}

Some pointing drift due to gyro control was observed, as anticipated. In particular the edge of the WFC3 field of view drifted onto the lunar disk and the last pairs of images were severely impacted by scattered light. This drift did allow us to confirm the orientation of the spacecraft field of view, however. A preliminary analysis of the data were reported at the Lunar and Planetary Science Conference (Storrs and Colaprete 2010). Sample WFC3 data is shown in Fig. 15. A possible slight enhancement of light in the WFC3 image immediately after impact (Fig. 15) may be evidence of an OH plume from the LCROSS impact, or it may be an instrumental artifact due to, for example, excess dark current at the corner of the chip. 
Fig. 15 HST image of the impact plume or instrumental artifact

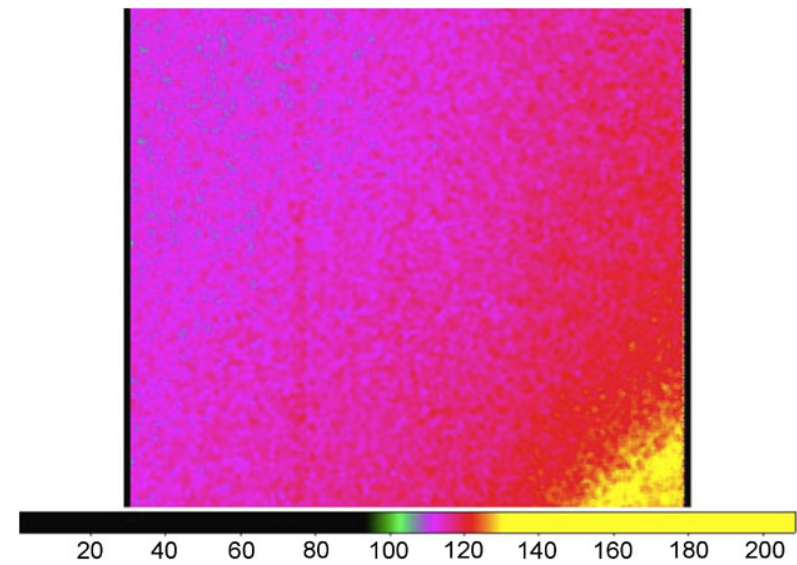

\section{Keck Observatory, Hawaii (Infrared Observations)}

\subsection{Planned Observations and Observing Techniques}

The observations of the LCROSS impact by Keck Observatory were executed in a coordinated effort with the Gemini Observatory, NASA Infrared Telescope Facility and Subaru Telescope. All four telescopes obtained imaging and spectral data sets exactly as planned. Synced video feeds from each of the telescopes to the mid-level facility on Mauna Kea allowed two team members to confirm pointing to the impact location by each of the four telescopes and to simultaneously witness the four experiments. The LCROSS observations required technical innovations from all four observatories to point and track on the site of the expected ejecta plume with sub-arc second precision, the result of concerted investment by observatory staffs to develop and test proof-of-concept solutions to the highly unusual observing requirements. Preliminary analysis indicates that the pointing fidelity at all sites was in the $0.05^{\prime \prime}$ to $0.3^{\prime \prime}$ range. Observations were obtained before, during and after the impact events to provide pre-impact baselines and the potential to analyze the impact plume evolution at 30-90 sec sampling cadence. The combined spectral coverage spanned 1.94.3 microns, with separate experiments designed to detect solid water-ice grains and water vapor in the ejecta plumes.

The LCROSS impact event was observed from the W. M. Keck Observatory using the NIRSPEC high-resolution long-slit spectrometer on the Keck II $10 \mathrm{~m}$ telescope. The goal of the observations was to detect three non-resonant fluorescent water vapor lines near 2.95 microns from levels near the ground-state so as to be sensitive to water molecules at 50-150

$\mathrm{K}$. The design of the experiment was simple because of the pointing and guiding constraints of the Keck telescope: pointing and guiding on the Moon are accurate after the guider has locked onto a bright peak or small crater. The field-of-view of the annular guider allowed for visual acquisition of the offset crater and impact position, and for shifting the offset crater and impact position into the slit and consequently out of view of the guider. Moving the telescope off the limb to "sky" required a different guider acquisition so that the pointing reference would be lost and have to be reacquired. Therefore, the observation setup was obtained and maintained through the two LCROSS impacts. 


\subsection{Pre-Impact Planning and Test Runs}

W. M. Keck Observatory obtained spectra with NIRSPEC at a resolving power of 27000, gathering data that encompasses the non-resonant fluorescent lines from water vapor near 2.95 microns. Prior to and following the impact, NIRSPEC SCAM images were obtained with the slit-viewing camera through the HeI filter enabling a confirmation of slit placement.

Starting more than an hour before impact the strategy was: point to the best Earth-view location of the LCROSS impact just above M1, the mountain in front of Cabeus, using offset coordinates from a small crater on the northern slope of M1 in sunlight; rotate the slit to be parallel to the ridge of M1; position the slit 2-3 pixels away from the ridge so that scattered light from the ridge is minimal in the slit and verify the guiding drift towards the ridge over 12 minutes does not introduce more scattered light into the slit; verify integration times for the observing conditions (maximize signal-to-noise and minimize read time) and verify timing of instrument macros to confirm the total duration of each integration plus read and store time and duration of eight consecutive integrations; then maintain guiding and data acquisition using macros to obtain a before-impact baseline.

\subsection{Impact Night Execution}

Four minutes before impact marked the start of an instrument macro of consecutive integrations with each integration being $90 \mathrm{sec}$ but taking $\sim 111 \mathrm{~s}$ to complete, so that the impact occurred about 18 seconds into the third of eight integrations. Through the Centaur impact, the data were acquired successfully, so a second set of eight integrations was started immediately in order to observe the S-S/C impact and about 3 minutes after that impact. A third set of 8 integrations was initiated to ensure any temporal evolution was captured. At the high resolving power of NIRSPEC $(R \sim 27,000)$, the telluric transmission was modeled to fit each spectrum so that the water vapor was derived for each spectral integration. This analysis technique allowed the pointing and tracking to remain locked onto the impact site. Following the impacts, sky measurements were made at lunar tracking rates at 30 arcsec and 300 arcsec to the South on the sky, off the lunar limb. By this time of the night, the airmass of the Moon was larger than at impact, so sky measurements also were obtained at sidereal rates at the same air mass as the Centaur impact. Then, flux calibrations were accomplished by obtaining spectra of a B star along with associated sky measurements. Following the completion of the experiment and flux calibration, we returned to the region of M1, mapping the scattered light on and off M1 to better characterize the scattered light contributions to the slit at different distances from the top of the mountain and to characterize the continuum spectrum of the lit terrain in front of M1.

\subsection{Summary of Technique}

The accuracy of pointing with Keck is good to a fraction of the slit width when the annular guider is locked onto a bright peak or small crater near the terminator. On impact night, the central crater of Cacetus $\mathrm{C}$ was used to lock onto lunar rates by observing support staff. When guiding on the impact position, viewing the impact site is inaccessible because the light path goes into the instrument through the central hole in the annular guider. The narrowest filter, the HeI filter (1.083 microns), was used with SCAM (the NIRSPEC slit viewing imager) to image the impact site and the positioning of the slit with respect to the ridge of M1. Since the spectral observations require a different filter, the K filter, these imaging observations could only be obtained when spectra were not being obtained. On the night 
before impact and the night of impact, the positioning of the Keck slit was verified by the two team members who were coordinating all four experiments from linked video screens at the mid-level facility at Mauna Kea. On the night of impact, the images through the HeI filter were saturated in the bright terrain but not saturated in the Cabeus Crater shadow, revealing gradations in the transitions between bright and dark. From these images, the gradations in scattered light varied in extent from the top of M1 as the seeing was fluctuating prior to and after the impact, which was confirmed by observations at the other Mauna Kea telescopes.

From impact plume models by Goldstein et al. (2008) and Summy et al. (2009), the column density for water vapor was predicted to be highest at intersection between the impact plume and the sun horizon, approximately $0.8 \mathrm{~km}$ above the impact point and behind M1. Due to the expanding plume, the column density was predicted to be the highest in the first tens of seconds. The earth viewing geometry allowed the plume to be observed at $\sim 2.5 \mathrm{~km}$ above the impact position, over the peak of M1, the mountain in front of Cabeus Crater. However, if the plume expanded laterally as expected, the plume possibly could be observed at lower altitudes along the (altitude-declining) flank of the mountain. Therefore, the Keck slit was positioned parallel to the ridge of M1, viewed on the sky as the East edge of M1.

\section{Magdalena Ridge Observatory, New Mexico (Ultraviolet-Visible-Infrared Observations)}

\subsection{Planned Observations and Observing Techniques}

Planned observations from Magdalena Ridge Observatory (MRO) were a set of optical CCD images. The primary observations planned were centered around the MRO 2.4-m telescope. This telescope uses an alt-az mount that is designed for high-speed pointing and tracking for following objects in low-Earth orbit. High-precision pointing and tracking made it possible to quickly setup on any lunar location in a matter of seconds. The system was also able to easily point and track relative to the trajectory of the impacting spacecraft.

To make the most of this facility a two-channel imaging system was used that was developed for observing Pluto stellar occultations. We used two Princeton Instruments PhotonMAX frame transfer CCD cameras mounted on a dichroic holder and mount. This system splits the nominal wavelength sensitivity range into roughly equal halves - UV to green and red to near infrared. The maximum array size is $512 \times 512$ pixels but can be windowed down to a smaller sub-array for a faster frame rate.

Raw sensitivity calculations indicated that the predicted impact properties would be detectible. If later predictions indicated a fainter plume our expectation was that we could use slower frame rates and still detect the impact debris. The primary perceived risk to this observation was that the impact would be significantly brighter than predicted and could thus lead to saturation.

To provide some measure of backup against saturating the prime experiment we planned to use a pair of portable 14-inch Schmidt-Cassegrain telescopes. One telescope was equipped with a third frame-transfer CCD using an older Princeton Instruments Micro-MAX camera with a choice of BVR or I filters. The second system was outfitted with an off-theshelf video camera. The difference in aperture and sensitivities of these systems gave us a factor of 30 insurance against a brighter-than-predicted observable. These telescopes have significantly poorer pointing and tracking capabilities than the primary telescope but these cameras had a field of view that was large enough to render the lower accuracy moot. 


\subsection{Pre-Impact Planning and Test Runs}

Two half nights were allocated and supported one month prior to the impact to practice acquiring the target location and run end-to-end tests of the telescopes and camera systems. Two half nights were allocated for the impact observations themselves - the night before and the night of the impact.

In addition, two half nights were scheduled one month post-impact in the event that additional observations were needed to complete the dataset (such as calibrations).

\subsection{Impact Night Execution}

Due to poor weather during the scheduled pre-impact runs, impact night was the first opportunity at MRO to obtain images of the Moon with the above described systems without interference from clouds. These images instantly revealed the primary and unexpected challenge for the observations: the nominal images from the system were saturated in the two-channel imaging observations with the 2.4-m telescope. Even with the smallest possible sub-array size for an effective frame rate of about 30 frames per second, the images were saturated by the illuminated portions of the Moon's surface. The dark shadowed area for the Cabeus impact site was overrun by charge bleeding over from the illuminated portions. With just hours to impact we had to find a work-around to reduce the flux from the Moon to prevent saturation. The solution we adopted was to insert a Bessel U filter into the beam ahead of the dichroic. The hope was that the known red-leak of a glass $U$ filter would be split from the $\mathrm{U}$ transmission and act like a dual-band neutral density filter. This might have worked if the dichroic had been built for a range shortward of 0.400 microns. Instead, from 0.400 microns down to the UV atmospheric cutoff the dichroic acted like a half-silvered mirror resulting in a nearly identical passband for each of the two imaging channels. This fact was not discovered until several days post-impact during data calibration activities.

A far more serious issue for these observations was the dynamic range required of the observations. In a circumstance (such as ours) where one must avoid saturating on the illuminated portion of the Moon, the signal-to-noise ratio is not simply an issue of raw detectivity. The ideal case would be to use a narrow band filter whose central wavelength and width would put the illuminated terrain just below saturation for the desired read-out time. It was estimated from the LCROSS data that the surface brightness of the ejecta plume was $100 \times$ lower than the illuminated terrain. Thus, a filter/exposure time combination that would give $90 \%$ of saturation $(65,000 \times 0.9 \mathrm{DN})$ would thus yield a signal of $650 \times 0.9 \mathrm{DN}$ on the plume. Reaching this condition requires a careful tweaking of the system throughput which is seldom possible with current filter technology. At MRO, the use of the Bessel U filter cut the illuminated surface flux to roughly $3000 \mathrm{DN}$ per frame. The per frame signal from the plume was then $30 \mathrm{DN}$ against the scattered light signal from the illuminated terrain. Coadding images can increase the raw detectivity but suffers from the inclusion of scattered light noise from atmospheric seeing variations. For our data we suffered from rather strong winds and strongly varying weather conditions and our seeing was not ideal ( $\sim 2 \operatorname{arcsec})$.

On impact night the target region on the Moon was acquired while it was only 10 degrees above the horizon. The team worked around clouds low on the horizon for a short time and then conditions became clear. Strong winds did emanate, however, out of the west. The MRO 2.4-m telescope was self-shielded by its dome for all the observations and did not suffer significantly from wind shake. The small telescopes, however, were set up on the ground outside the building and were shielded by only a truck set to block as much wind as possible. Unfortunately, the wind shake was so severe that no useful data were collected with the small telescopes. 


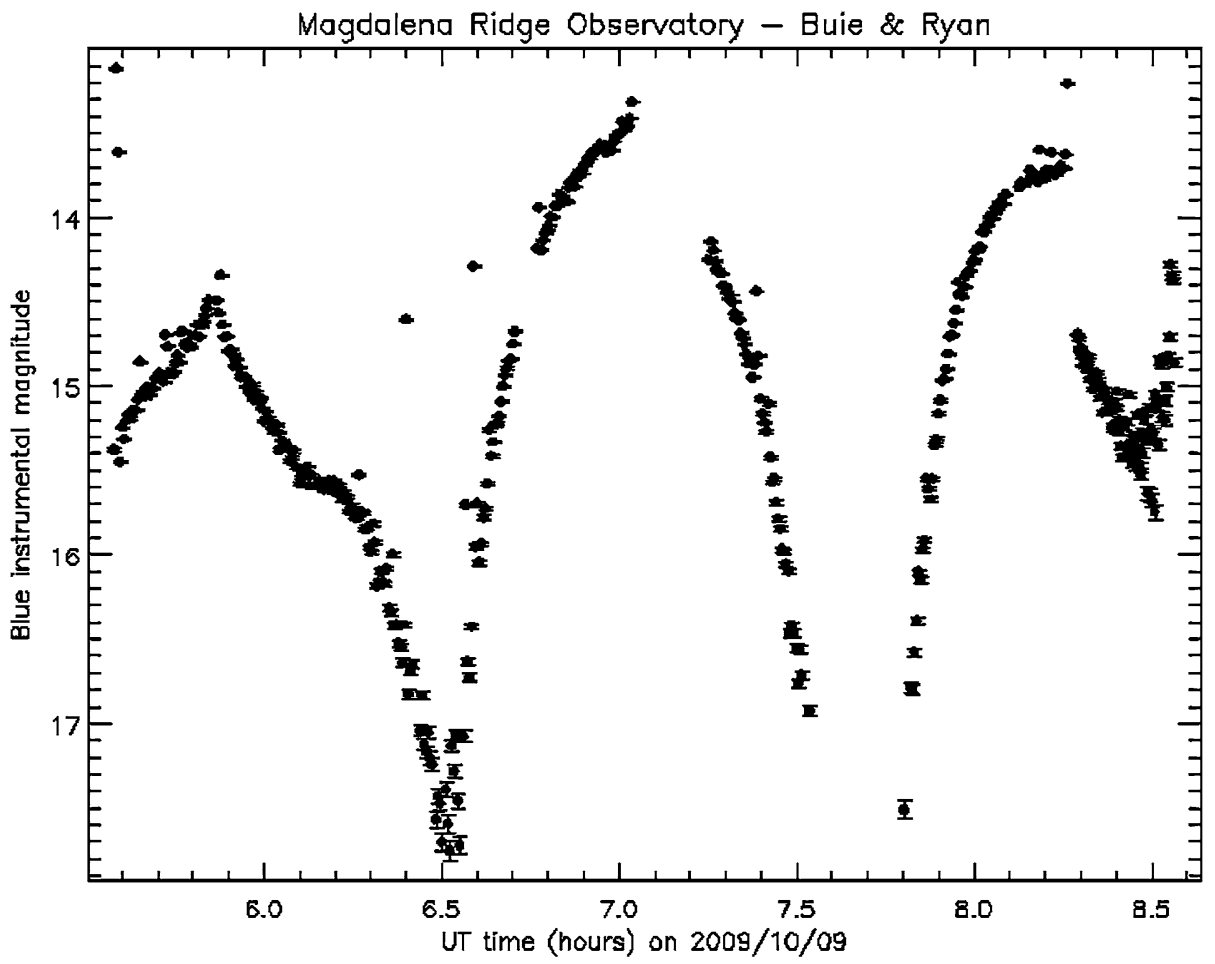

Fig. 16 Light curve of the LCROSS Centaur obtained at Magdalena Ridge Observatory

After Moon rise but prior to impact a series of imaging observations were collected to monitor the brightness of the Centaur upper stage which was easily detected by the fullthroughput 2-band setup. Figure 16 shows the blue lightcurve of the Centaur booster up until the time where glare from the nearby Moon made it impossible to collect useful images. The data cannot be accurately corrected for extinction but the lightcurve is so large that this deficiency is minimal. The lightcurve shows clear indicates of rotation with a principal rotational component with a period of roughly two hours (assuming a 2-max/2-min lightcurve structure). The brightness varies by more than six magnitudes ranging at its faintest to a brightness that was barely detectable even with dynamically adjusting the exposure time to compensate. The lightcurve shape evolves during the observations and if this were a natural object one would quickly conclude that it was exhibiting non-principal axis rotation. In this case the data indicates that the booster is tumbling which is not unexpected.

The data set is archived in NASA's Planetary Data System (PDS) and is available for download by the general public (Buie 2010a, 2010b).

\subsection{Summary of Technique}

Several suggestions for an "ideal" imaging system can be gleaned from the MRO experiences in observing the LCROSS events. A significantly faster camera (more than a factor of 100) would be advantageous to use all the light collected by the 2.4-m telescope. However, this fast camera must also not suffer high readout noise (more than a few DN) since many frames would need to be coadded to obtain a useful signal on the ejecta. Also, the camera 
would still need to have a large dynamic range A/D (at least 16 bits) to collect all of the relevant information.

\section{MMT Observatory, Arizona (Visible-Infrared Observations)}

\subsection{Planned Observations and Observing Techniques}

The observations designed for the MMT 6.5-m telescope and equipment had two scientific goals:

(1) Test for the presence (or absence) of phyllosilicates in the lunar soil, formed by the action of aqueous alteration (the alteration of material by the interaction of that material with liquid formed by the melting of incorporated ice), as an indicator of the presence of water ice trapped near the lunar pole. A primary indicator of aqueous alteration is the 3.0 micron water of hydration absorption feature attributed to structural hydroxyl $(\mathrm{OH})$ and interlayer and adsorbed water $\left(\mathrm{H}_{2} \mathrm{O}\right)$ in phyllosilicates. A weak spectral absorption feature centered near 0.7 micron, attributed to an $\mathrm{Fe}^{2+} \rightarrow \mathrm{Fe}^{3+}$ charge transfer transition in oxidized iron in phyllosilicates, has served as an indicator of the 3.0 micron feature in reflectance spectra of "grey" asteroids and established a lower limit on the presence of aqueous alteration in these asteroids (Rivkin et al. 2002). Recent spacecraft data have identified the 3.0 micron feature on the lunar surface (Pieters et al. 2009; Sunshine et al. 2009; Clark 2009). A search for the 0.7 micron feature in broadband spectral images taken by Galileo during its first Earth-Moon fly-by suggest that it is present near the lunar south pole (Vilas et al. 2008). Hydrogen content in the soil can be established from knowledge of the depth of the 3.0 micron absorption feature (Rivkin et al. 2003). Thus, evidence obtained concurrently for these two features in the plume material excavated as a result of the impacts would strongly suggest the presence of water having affected the lunar surface mineralogy in a deeply shadowed crater.

(2) Constrain knowledge about the structural evolution of the plume with time following the impacts, which is governed partially by amount of water vapor present in plume material (Heldmann et al. 2007).

These goals directly address the stated science goals of the LCROSS mission.

The highest spatial resolution at the MMT, $2.11 \mathrm{arcsec} / \mathrm{mm}$, is provided with the $\mathrm{f} / 15$ secondary having a deformable mirror that is used with a natural guide star adaptive optics (AO) system. We aimed to address these science goals by first obtaining spectra of the plume material across the wavelength range of 2.5-4.5 microns using the Clio two-dimensional imager and spectrograph (Freed et al. 2004). Within the f/15 natural guide star adaptive optics top box, a beamsplitter directs light $<0.95$ micron to a CCD47 camera sensitive to a lower wavelength range. Using a filter centered near 0.7 micron with a passband of 0.040 microns, the presence and development of the plume near 0.7 micron could be imaged. Significant brightening of the plume dust material with time was expected in this region (Heldmann et al. 2007). The AO system would configure the f/15 secondary using a bright star in the vicinity of the Moon. The secondary would then be frozen in that configuration, in order to optimize the seeing in the area of the Moon for these observations.

\subsection{Pre-Impact Planning and Test Runs}

Six months prior to the LCROSS impact, we started scheduled observations. At this time, we reviewed the observing procedure that we proposed and the available instrumentation 
Fig. 17 CLIO slit position at MMT Observatory

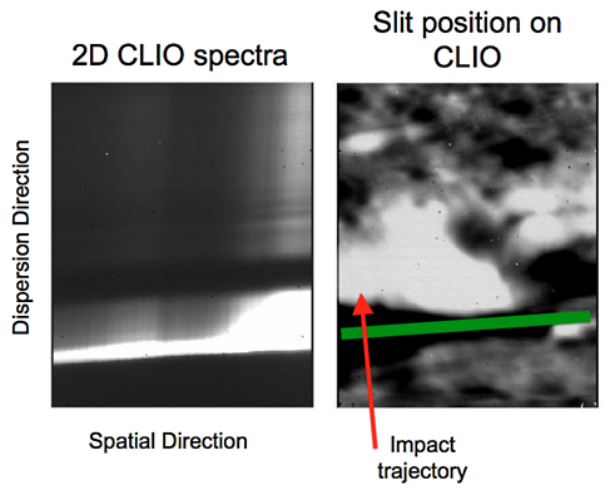

that we could access. We conducted a test of the AO system to experiment with whether we were able to close the loop on the lunar surface. The AO system worked on the lunar surface within the visible disk, but did not work near the edge of the Moon. Hence, we stayed with our initial strategy of closing the loop on a nearby bright star, freezing the secondary mirror in its corrected position in order to compensate partially for atmospheric turbulence.

We also scheduled two full nights of observing prior to the LCROSS impacts. One night was scheduled a month in advance in September 2009, in order to observe the Moon during the identical lighting conditions as the night of the LCROSS impacts. The night before the LCROSS impacts was also scheduled for testing the procedures and instrumentation. Both of these nights were completely lost to clouds.

\subsection{Impact Night Execution}

Observing conditions on UT 9 October 2009 were clear and photometric with seeing of 0.38 arcsec derived from wavefront sensing. We slewed the telescope to the Moon and found Cabeus Crater, located the position where the impact was directed into the surface of the Moon, and took calibration data on the area around the Moon. All equipment was tested to ensure that it was working correctly, and that data were being recorded.

Approximately 1-2 hours before the first impact, we collected data on two A0 IR calibration stars (HD22859, HD43607), and the solar analogue star Hyades 64 (HD 22089). We then locked on to a bright star near the Moon with the AO secondary active, and froze the mirror into configuration. We moved to Cabeus Crater and aligned the slit for the Clio spectrograph to emphasize the shadowed portion of Cabeus Crater parallel to the N edge of the crater, thus ensuring that any plume growth that was visible would be seen through the slit as the plume grew (Fig. 17). The CCD47 was co-aligned on the same area of the slit. We observed from 8 minutes before the first impact to approximately 36 minutes after the second impact when twilight limited our continuing the observations.

The data set is archived in NASA's Planetary Data System (PDS) and is available for download by the general public (Vilas 2010a, 2010b, 2010c).

\subsection{Summary of Technique}

The primary objective of these observations was to identify signatures of aqueous alteration in lunar reflectance properties as an indicator of the presence of water ice trapped near the lunar south pole. The presence, shape and spectral placement of a diagnostic feature near 3.0 micron coupled with the growth of the plume in a 0.7 micron passband, were sought by 
the experiment. A two-dimensional IR spectrograph coupled with a visible imaging camera both having a high temporal readout both concurrently observed Cabeus Crater during the impacts.

\section{Mount Lemmon (Arizona, Visible Observations)}

\subsection{Planned Observations and Observing Techniques}

We attempted LCROSS lunar impact observations at LOAO (Mt. Lemmon Optical Astronomy Observatory) for CCD imaging. The main purpose of the imaging observation was to measure the total energy released by impact flash which is directly related to the luminous efficiency. We adopted a fast drift scan technique for the LOAO 1 meter remote telescope equipped with Kodak 2K CCD.

\subsection{Pre-Impact Planning and Test Runs}

We successfully performed several drift scan practices for LOAO. After a series of repetitive test observations we determined proper exposures for different target areas.

\subsection{Impact Night Execution}

At LOAO, the telescope was drifted West-to-East with a speed of 100 pixels per second, which is equivalent to $64 \mathrm{arcsecond} / \mathrm{sec}$ for both the Centaur and S/S-C impacts. We expected at least 6 pixels of streak at CCD images assuming the shortest flash duration of 0.06 second (Heldmann et al. 2007; Shuvalov and Trubetskaya 2008). Slewing the telescope started on $T-8$ seconds and exposure started on $T-2.5$ seconds. Light travel time of the impact flash from the Moon was compensated for by internet delay due to remote operation of the telescope via the internet.

\subsection{Summary of Techniques}

Drift scan has been successfully implemented yet detection of impact flash was not possible due to the geometry of the impact point.

\section{Mount Wilson, California (Infrared Observations)}

\subsection{Planned Observations and Observing Techniques}

Mount Wilson is in the San Gabriel Mountains of the Angeles National Forest of Southern California, approximately 33 miles from downtown Los Angeles. The plan at Mount Wilson was to observe dynamics of the LCROSS impacts and ejecta in the near and shortwave infrared at $30 \mathrm{~Hz}$ frame rate using the 60-inch telescope and a high performance infrared camera. Infrared video data were transmitted by line-of-sight microwave link from the telescope floor to the Carnegie Observatories office in Pasadena, CA, using a Premier Wireless model AG-6001 wireless ethernet system. From there, the data were sent by Internet to the LCROSS team at NASA Ames. Surface brightness estimates for the impacts and ejecta plumes were made based on A0 V and G2 V star calibration measurements and by comparison with well characterized parts of the lunar surface. 
The Mount Wilson 60-inch is the largest telescope in the world devoted entirely to public viewing of astronomical objects. It was the largest telescope in the world from its first light in 1908 until completion of the Mount Wilson 100-inch telescope in 1918. The telescope is a bent-Cassegrain reflector with a 60 -inch diameter primary mirror, a focal ratio of $\mathrm{f} / 16$ and focal length of 960 inches or $2438 \mathrm{~cm}$.

A $1280 \times 1024$ pixel format infrared camera was operated at the Cassegrain focus of the 60 -inch telescope. This high performance digital camera with InGaAs detectors is sensitive to light in the $0.9-1.7$ micron spectral region and was operated at room temperature without any spectral filter. Each detector in the camera's array is 20 microns by 20 microns and subtends $0.82 \mu \mathrm{rad}(\sim 0.2 \mathrm{arcsec})$ in each angular dimension at the 60 -inch Cassegrain focus. Camera field of view on the 60 -inch telescope is $1050 \mu \mathrm{rad} \times 840 \mu \mathrm{rad}(218 \operatorname{arcsec} \times$ $174 \mathrm{arcsec})$. The camera is similar to the NIR cameras that were in the LCROSS payload.

\subsection{Pre-Impact Planning and Test Runs}

A Pre-Impact test run occurred on the night of 2009 October 6-7. All elements of the observing system were tested, including the microwave link. Earlier test runs planned for late August through September did not occur because the Station Fire, which devastated 160,577 acres in the Angeles Forest and threatened Mount Wilson Observatory several times, prevented safe access to the observatory. The Mount Wilson LCROSS team required special permission from the California Department of Transportation to gain access to the observatory during this test run and for impact night.

Overall, the test run was successful. Seeing varied from about 2 arcsec at the beginning of the night to about 1 arcsec around midnight. The seeing deteriorated to about 2 arcsec after about 0200 PDT on the 7 th. Plans were made to minimize possible seeing effects from equipment and operators by moving equipment on the telescope floor away from the LCROSS impact telescope line of sight. To avoid saturation of the bright lunar image, the camera was operated with an integration time of about $250 \mu \mathrm{sec}$, much less than the $33 \mathrm{msec}$ frame period.

Position encoders on the telescope behaved very erratically during the test run, which made it difficult to point the telescope well enough to put calibration stars inside the field of view of the camera. In some cases, calibration stars were located by removing the camera and putting an eyepiece into the system and then centering the star in the eyepiece. However, removing the camera from the telescope put the camera at some risk from handling and changed the overall spatial alignment of the camera-telescope system, which made it a very unattractive operating procedure. Given this type of problem had not been seen previously, the Mount Wilson staff felt that electromagnetic interference (EMI) from the camera might be affecting the encoders. To reduce possible effect of camera emissions on the telescope encoders, EMI chokes were put into the system for LCROSS impact night.

\subsection{Impact Night Execution}

The infrared camera was setup on the 60 -inch telescope shortly after sunset on 8 October 2009. Conditions were good with photometric conditions at sunset and smog below the observatory. Sky conditions remained fairly good during and after the impact, although seeing deteriorated roughly $30 \mathrm{~min}$ before Centaur impact from what it had been earlier in the night. Seeing remained at 1-2 arcsec during the impact events.

Camera system performance was monitored throughout the evening by observing stars and other astronomical objects. The camera field of view was navigated across the lunar disk 
and many test videos of the impact site were collected before the first impact. Test videos were transmitted to NASA Ames at about 40 minutes before impact and processed video of the impact times was sent to NASA within 60 minutes of first impact. The optimum camera integration time was found to be $315 \mu \mathrm{sec}$, approximately $26 \%$ longer than the integration time used during the test run. The telescope encoder problems encountered during the preimpact test run persisted on impact night, despite the EMI chokes installed in the camera. The encoder problems did not affect ability to locate the impact site.

\subsection{Summary of Technique}

The Mount Wilson observing run was successful in that all equipment worked as expected and continuous infrared video of the predicted impact location was collected before, during and after the predicted impact times. Roughly 23 minutes (41199 frames) of video with 108 GB of raw data were collected.

\section{NASA IRTF, Hawaii (Infrared Observations)}

\subsection{Planned Observations and Observing Techniques}

Observations of the LCROSS impacts with the NASA Infrared Telescope Facility (IRTF), a $3 \mathrm{~m}$ telescope located at the Mauna Kea Observatory, used the SpeX instrument, a facility class low- to medium-resolution near-infrared spectrometer (Rayner et al. 2003). The goal of these observations was to record 2 to 4 micron spectra of the impact ejecta before and immediately following each of the LCROSS impacts to constrain the amount and spectral shape of light scattered by material excavated from the floor of Cabeus Crater. Simultaneously with the spectra, we planned to save images of the surrounding terrain with the SpeX guide camera. This near-infrared camera images a $60^{\prime \prime} \times 60^{\prime \prime}$ field-of-view surrounding the SpeX slit simultaneously with the spectrometer integrations. The sun-lit Moon is very bright in the near-infrared, even for the SpeX guide camera, which has a minimum integration time of $0.12 \mathrm{~s}$. For imaging, we chose a narrowband filter centered at 2.294 microns with a spectral width of $1.5 \%(\mathrm{CO} v=2 \rightarrow 0)$. Additional attenuation was provided by a near-infrared neutral density filter with a transmission of $1 \%$. This combination of filters allowed us to integrate up to a few seconds without saturating on bright lunar terrain.

We chose to observe the Moon with SpeX's $0.8^{\prime \prime}$ wide by $15^{\prime \prime}$ long slit. Line emission and absorption in Earth's atmosphere typically dominates 2 to 4 micron spectra of astronomical targets. The atmospheric seeing on the night of impact was approximately $0.6^{\prime \prime}$, therefore the spectral resolution of unresolved sources was seeing limited, but extended sources (such as smooth lunar terrain) were slit limited. Our choice of an $0.8^{\prime \prime}$ wide slit enabled the collection of more light although the use of larger slits also increases the number of background and sky photons. Ultimately, photons from the bright background and sky limits our integration times, duty cycle, and signal-to-noise ratios. Choosing the narrow $0.8^{\prime \prime}$ SpeX slit had profound implications on our observing strategy, demanding a telescope pointing accuracy of $0.4^{\prime \prime}$ (half the slit width), which in turn required that our knowledge of the lunar terrain and the location of the impact site on a common lunar coordinate grid be at least as accurate.

\subsection{Pre-Impact Planning and Test Runs}

In order to verify that the IRTF could deliver sub-arcsecond pointing near the south pole of the Moon, we developed a technique to locate the precise topographic location of the 
impact site. Through a combination of the Jet Propulsion Laboratory's HORIZONS tool (Giorgini et al. 1996), lunar topography maps, and images of the Moon from the Consolidated Lunar Atlas (Kuiper et al. 1967), and from the IRTF, Gemini, Keck, and Apache Point practice runs, we identified a sequence of craters on which to center the SpeX slit. Developing this sequence was a challenging process, primarily due to the observing conditions near the south pole of the Moon. Here, the terrain is foreshortened and self-obscuring due to the projection of the lunar ellipsoid and topography onto the telescope's image plane. Furthermore, the orbit and libration of the Moon changes the terrain orientation from night to night and month to month, which has dramatic effects near the south pole. Topographic maps and lighting models (scene descriptors) helped us identify craters and other features that would be visible in images from the ground, and have angular sizes large enough to be identifiable after the effects of atmospheric blurring are taken into account (the seeing at the IRTF in the K-band is typically 0.5 to $1^{\prime \prime}$ ). Each crater in our final sequence was chosen to provide successively greater pointing precision with successively smaller distances to the impact location. The coordinates and altitudes of these craters were determined from the topographic maps of the South Polar region. The final crater before moving to the impact location was located at $328.1^{\circ} \mathrm{E},-82.2^{\circ} \mathrm{S},-1.1 \mathrm{~km}$, with an apparent diameter of $\approx 2.4^{\prime \prime}$ $(4.4 \mathrm{~km})$. We used HORIZONS to transform the lunar longitudes, latitudes, and altitudes into Right Ascension and Declination, then with a simple script we generated plane-of-sky offsets between craters. Thus, the telescope could use the non-sidereal tracking rates of a nearby crater (e.g., Casetus), and the observers could offset differentially from crater to crater.

At the IRTF, we quickly realized that guiding on the Moon would be crucial to keeping our slit centered on the target. At first, we used manual tracking in our test observing sequences, but later we successfully tested auto-guiding on locally bright features (e.g., mountain peaks and sun-lit crater walls) in the surrounding terrain. The SpeX auto-guide routine employed a centroid algorithm to center on the region of interest. On the night of the impacts, auto-guiding was enabled and the telescope tracking rates were set to follow the Moon. During the impacts, the telescope position was held within 1 pixel of our target position ( $68 \%$ of the time) with a maximum deviation of 2 pixels $\left(1\right.$ pixel $=0.12^{\prime \prime} \approx 220 \mathrm{~m}$ ).

The ejecta plume was expected to be initially obscured by mountains at the limb of Cabeus Crater. Eventually, the plume would reach sunlight, and continue to climb until it became visible to ground-based observers. Since the surface brightness of the plume would be greatest near the impact site, we were driven to place the SpeX slit on the point at which the plume is immediately visible to the IRTF. However, atmospheric blurring and guiding jitter would scatter photons from the bright mountains into the slit. These effects increase the observed background and decrease the resulting signal-to-noise. To mitigate this issue, we placed the slit one slit width $\left(0.8^{\prime \prime}\right)$ away from the mountain ridge (Fig. 18). The distance was primarily driven by the measured seeing $\left(0.6^{\prime \prime}\right)$ and the increased field-of-view inside the shadowed crater, allowing us to potentially measure some horizontal evolution of the plume.

\subsection{Impact Night Execution}

On the night of impact, we practiced our observing procedure twice to verify that our integration times and human reaction times were adequate. The observing procedure was as follows: 1) locate the target site using our pointing sequence and begin guiding on a nearby bright feature (Fig. 18); 2) at 5.0 minutes before impact start a sequence of integrations with the spectrometer that would last for 6.5 minutes; 3 ) nod the telescope to record a sky 
Fig. 18 The IRTF SpeX guide camera image of the slit at the time of the LCROSS Centaur impact. The slit $\left(0.8^{\prime \prime} \times 15^{\prime \prime}\right)$ has been outlined in white for clarity

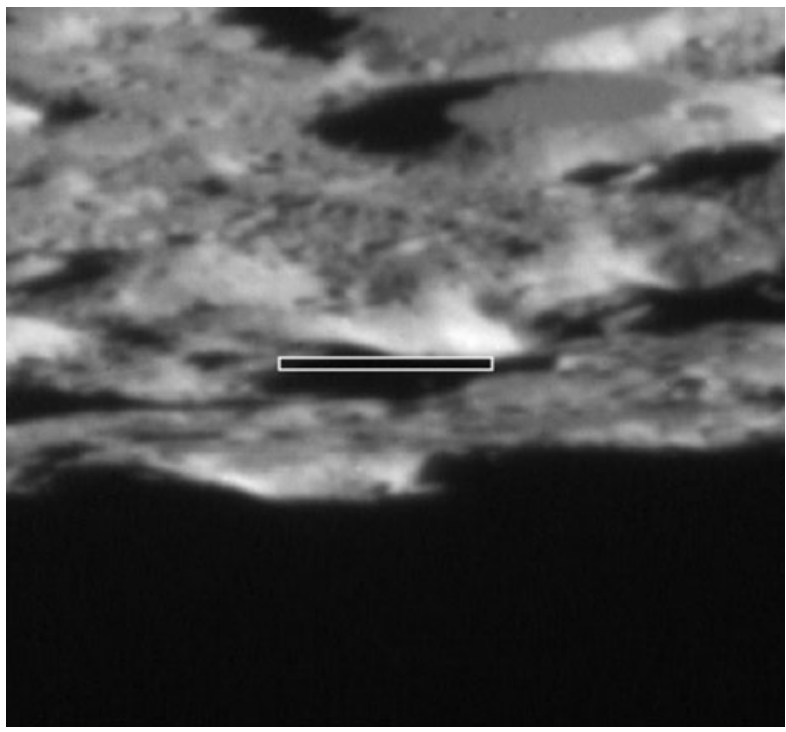

frame off the lunar limb; 4) return to the target and re-engage auto-guiding; 5) at 3.5 minutes after impact (30 seconds before S-S/C impact), begin another set of integrations spanning 2.0 minutes total; and, 6) nod the telescope for another sky frame. The SpeX guide camera was set to save the auto-guiding images at all times. The integration times and duty cycle of the spectrometer were carefully chosen so that one integration/read out/save cycle would take 15 seconds to complete. This effort allowed us to begin the observations in advance of the impacts, and allowed us to monitor the situation for telescope issues and the latest updates from the LCROSS Science Operations Center. At the time of impact the procedure was executed as planned.

\subsection{Summary of Technique}

The IRTF SpeX LCROSS impact data presents many reduction and analysis challenges of which background subtraction is a primary issue. Few, if any, of the 5 minutes of pre-impact spectra adequately removed the backgrounds from the post-impact spectra. The telescope jitter, seeing, and transparency variations (thin cirrus were present over Mauna Kea) were great enough that after background subtraction, some residual light scattered from the nearby terrain was always present at the target location. The residuals provide a systematic error to our observations. If an LCROSS-like impact mission were to be repeated, the observations would require mitigation techniques to enhance the data robustness. One possible approach would be to obtain a very accurate spectral scan over a large region around the impact coordinates, which could be cropped to the exact location of the slit at any time.

\section{Subaru Telescope, Hawaii (Infrared Observations)}

\subsection{Planned Observations and Observing Techniques}

Planned observations at the Subaru Telescope included high-resolution near-IR spectroscopic observation of the LCROSS impact ejecta plume. The observations targeted water 
emission lines near wavelengths of 2.9 microns in nonresonance fluorescence bands (hot bands) because these emission lines are absorbed much less efficiently by atmospheric water vapor than fundamental water band emission. Infrared spectra were collected with the Infrared Camera and Spectrograph (IRCS) Echelle spectrometer at Subaru Telescope. The Echelle has a 1024-1024 pixel array detector, and these observations used a 170 pixel [9.37"]-4 pixel [0.27"] slit, corresponding to $18.7 \mathrm{~km}$ length and $0.54 \mathrm{~km}$ width, which leads to the spectral resolution $\lambda / \Delta \lambda \sim 10,000$.

Near-IR imaging observation with K' band using the slit viewer was also employed. This observation was intended to detect dust particles contained within the ejecta plume. The adaptive optics (AO) system was planned to be used in order to minimize any fluctuations in the field of view.

\subsection{Pre-Impact Planning and Test Runs}

The Subaru telescope conducted a couple of very short test runs before the impact as a part of engineering development activities.

\subsection{Impact Night Execution}

Both Echelle spectroscopic observations and slit viewer imaging observations with $\mathrm{K}^{\prime}$ band using the AO system were collected as planned. The slit was placed on the permanently shadowed region in Cabeus Crater, parallel to the top of the Cabeus rim in order to place the impact plume in the middle of the slit. The Subaru adaptive optics (AO) system reduced wobbles of the slit within $\pm 0.05^{\prime \prime}(\sim 0.1 \mathrm{~km})$ during the observation. Time variation of infrared spectra were obtained before and after the Centaur and the Shepherding Spacecraft (S-S/C) impacts. The exposure time of the IR spectra was 40 seconds. All data were successfully collected and no off-nominal situations were encountered.

\subsection{Summary of Technique}

The AO system suppressed fluctuation of the slit position down to only one pixel ( $\sim 100 \mathrm{~m}$ on the lunar surface). The spectral resolution of the Echelle spectroscopic observation was about 10,000 . The observing techniques were successful at collecting the planned data of the LCROSS impact events.

\subsection{Ground Based Observation Summary}

No ground based observatory has reported unambiguous detections of the LCROSS dust plume. Here we explore several possibilities to explain this lack of detection.

Based on data from the LCROSS Shepherding Spacecraft from the nadir-looking S-S/C UV-visible spectrometer, the visible peak flux of the dust plume occurred approximately 20 seconds after Centaur impact and peaked at $\sim 10 \mathrm{~W} / \mathrm{m}^{2} / \mathrm{micron} / \mathrm{str}$ (Heldmann et al. 2010). A combination of LCROSS Spacecraft data analysis, impact modeling, and laboratory data indicates that the LCROSS plume was composed of both a high angle and low angle plume component (Schultz et al. 2010; Heldmann et al. 2010). The high angle plume was narrow, reached an altitude on the order of $100 \mathrm{~km}$, and persisted for the entire $\sim 4$ minute descent of the S-S/C after Centaur impact. The low angle plume reached an altitude on the order of $15 \mathrm{~km}$ and persisted in sunlight for only $\sim 50$ seconds before the particles returned below the sun horizon. The brighter low angle plume thus reached altitudes where it should have been 
in the field of view of ground-based assets. The low angle plume, however, was significantly brighter than the high angle plume and primarily accounted for the peak flux at $\sim 20$ seconds after Centaur impact. The low angle plume was on the order of 100 times fainter than the nearby lit lunar terrain.

Based on this information, we expected that the LCROSS dust plume should have been observable by the ground based observatories. However, the lack of detection could be explained by several possible reasons. First, the morphology of the plume may have been such that the brightness when viewed from above (S-S/C vantage point) resulted in a brighter plume than when observed from the side (Earth-based vantage point). Another possibility is that if the ground-based visible imaging was not saturating on the lit lunar surface, then the 100 times fainter plume may not have been detected because the plume was below the threshold limits for the non-saturated lunar surface case. Saturating on the Moon can be problematic, though, as bleeding into the region of Cabeus where the plume was expected would also result in a null detection. Such factors highlight the complications in this type of observation. Regarding the near-infrared data, the LCROSS S-S/C observations from the near-infrared spectrometer show a brightness 2.5 times fainter than expected due to the presence of very small (submicron) dust particles in the plume. The lower flux in the near-infrared could lead to more difficult detection at these wavelengths from the ground. Due to the complicated nature of these observations and the subsequent data analysis, the Astronomer teams continue to analyze the data to carefully search for any signal from the LCROSS impact events.

\section{Conclusions}

The LCROSS impact events provided a unique opportunity to observe dual impacts on the Moon through a coordinated Observation Campaign. The LCROSS Observation Campaign utilized the expertise of a variety of different groups ranging from mission management, mission planners, science team members, orbital dynamics analysts, telescope operations, and the Astronomers themselves. The LCROSS Observation Campaign demonstrated the importance of including the Astronomers as official members of the LCROSS Science Team in order to enable effective and timely communications from the LCROSS Project to the Astronomer teams and vice versa. This experience also emphasized the importance of readiness tests and conducting practice runs to test out systems, equipment, and communications between and amongst the Astronomer teams and the LCROSS Science Operations Center. Incorporating a variety of observing assets into the Campaign helped ensure that a robust set of observations would be collected spanning a variety of observing vantage points and instrument types. The LCROSS Observation Campaign represents a successful collaboration of multiple teams and facilities distributed around the globe which enabled simultaneous observations of the LCROSS impacts from a multitude of observing assets.

Acknowledgements The LCROSS mission was funded by NASA's Exploration Systems Mission Directorate (ESMD) at NASA Headquarters. The authors gratefully acknowledge NASA's ESMD and the LCROSS Project Office at NASA Ames Research Center for managing the LCROSS mission. NASA's Science Mission Directorate also supported the LCROSS Astronomer Workshop and science analysis of the data. The Lunar and Planetary Institute (LPI) provided logistical support for both the LCROSS Site Selection and Astronomer workshops. NASA worked with USRA to provide support to several Astronomer teams for data collection, analysis, and data delivery to the Planetary Data System. In particular we thank Susie Slavney and Ed Guinness for assistance with archiving the ground-based observations within the Planetary Data System. We also thank each of the observatories and all of the individuals that supported the LCROSS Observation Campaign.

The AEOS team is grateful to B. Hema and E. Agader, the telescope operators at AEOS. This work was partially supported by the Independent Research and Development program of The Aerospace Corporation. 
This work is based on data from the Maui Space Surveillance System, which is operated by Detachment 15 of the U.S. Air Force Research Laboratory's Directed Energy Directorate.

The NMSU-NASA/MSFC team acknowledges the Universities Space Research Association, who supported the APO observations through contract number 03450-32. The NASA/MSFC team acknowledges partial support from the NASA Meteoroid Environment Office.

The MMT Observatory is a joint facility of the University of Arizona and the Smithsonian Institution.

The following individuals provided important support for the pre-impact test run and LCROSS impact observations from Mount Wilson: Brian Day, Tom Meneghini, Richard Bell, Rick Baldridge, Michael Kirkpatrick, Hannah Jorgensen, Sherri Reznik, Kim Bunnell, Nolan Andreasen and Ariel Bluy.

NASA Education and Public Outreach (EPO) provided funding to Mount Wilson Institute for use of the 60-inch telescope during the pre-impact test run and LCROSS impacts. Raytheon donated use of the $1280 \times 1024$ pixel format infrared camera used at Mount Wilson. Premiere Wireless donated use of an AG6001 wireless ethernet system operating in FCC approved bands from 4910 to $5825 \mathrm{MHz}$ to enable real time transmission of video data from Mount Wilson to the LCROSS team at NASA Ames.

IRAF is distributed by the National Optical Astronomy Observatory, which is operated by the Association of Universities for Research in Astronomy (AURA) under cooperative agreement with the National Science Foundation.

Gemini Observatory is operated by the Association of Universities for Research in Astronomy, Inc., under a cooperative agreement with the NSF on behalf of the Gemini partnership: the National Science Foundation (United States), the Science and Technology Facilities Council (United Kingdom), the National Research Council (Canada), CONICYT (Chile), the Australian Research Council (Australia), Ministério da Ciência e Tecnologia (Brazil) and Ministerio de Ciencia, Tecnología e Innovación Productiva (Argentina). C.E.W. and D.E.H. also acknowledge support from the NSF (AST-0706980), and NASA through a grant to SWRI.

Support for Hubble Space Telescope observations (program number HST-GO-11806.02-A) was provided by NASA through a grant from the Space Telescope Science Institute, which is operated by the Association of Universities for Research in Astronomy, Incorporated, under NASA contract NAS5-26555. Many thanks to Tony Roman for help at all stages of planning and implementation of these observations.

The LCROSS Project also thanks the NASA Goddard Space Flight Center Scientific Visualization Studio for visualizations of the Moon as viewed from Earth.

Open Access This article is distributed under the terms of the Creative Commons Attribution Noncommercial License which permits any noncommercial use, distribution, and reproduction in any medium, provided the original author(s) and source are credited.

\section{References}

J.R. Arnold, J. Geophys. Res. 84 (1979). doi:10.1029/JB084iB10p05659

M.W. Buie, Magdalena Ridge Observatory $2.4 \mathrm{~m}$ PHOTDOC Observations of LCROSS, NASA Planetary Data System, EAR-L-MRO24M_PHOTDOC-2-EDR-LCROSS-V1.0 (2010a)

M.W. Buie, Magdalena Ridge Observatory 2.4m PHOTGJON Observations of LCROSS, NASA Planetary Data System, EAR-L-MRO24M_PHOTGJON-2-EDR-LCROSS-V1.0 (2010b)

D.B.J. Bussey, P.G. Lucey, D. Steutel, M.S. Robinson, P.D. Spudis, K.D. Edwards, Geophys. Res. Lett. 30 (2003). doi:10.1029/2002GL016180

N.J. Chanover, Apache Point Observatory 3.5m Agile Observations of LCROSS, NASA Planetary Data System, EAR-L-APO3.5M_AGILE-2-EDR-LCROSS-V1.0 (2010)

R. Clark, Science 326 (2009). doi:10.1126/science.1178105

A. Colaprete, P. Schultz, J. Heldmann, M. Shirley, K. Ennico, B. Hermalyn, D. Wooden, W. Marshall, A. Ricco, R. Elphic, D. Goldstein, D. Summy, G. Bart, E. Asphaug, D. Korycansky, D. Landis, L. Sollitt, Science (2010). doi:10.1126/science. 1186986

D.H. Crider, R.R. Vondrak, J. Geophys. Res. 108 (2003). doi:10.1029/2002JE002030

W.C. Feldman, S. Maurice, A.B. Binder, B.L. Barraclough, R.C. Elphic, D.J. Lawrence, Science 281 (1998). doi:10.1126/science.281.5382.1496

W.C. Feldman, S. Maurice, D.J. Lawrence, R.C. Little, S.L. Lawson, O. Gasnault, R.C. Wiens, B.L. Barraclough, R.C. Elphic, T.H. Prettyman, J.T. Steinberg, A.B. Binder, J. Geophys. Res. 106, 23,231-23,251 (2001)

M. Freed, P.M. Hinz, M.R. Meyer, N.M. Milton, M. Lloyd-Hart, Proc. SPIE 5492 (2004)

J.D. Giorgini, D.K. Yeomans, A.B. Chamberlin, P.W. Chodas, R.A. Jacobson, M.S. Keesey, J.H. Lieske, S.J. Ostro, E.M. Standish, R.N. Wimberly, Bull. Am. Astron. Soc. 28 (1996)

D. Goldstein, D. Summy, A. Colaprete, P.L. Varghese, L.M. Trafton, Bull. Am. Astron. Soc. 40 (2008) 
J.A. Hackwell, et al., Proc SPIE 1235 (1990)

J. Heldmann, A. Colaprete, D. Wooden, E. Asphaug, P. Schultz, C.S. Plesko, L. Ong, D. Korycansky, K. Galal, G. Briggs, Bull. Am. Astron. Soc. 38 (2007)

J.L. Heldmann, A. Colaprete, D. Wooden, E. Asphaug, P.H. Schultz, C.S. Plesko, L. Ong, D. Korycansky, K. Galal, Lunar. Planet. Sci. Conf., Abstract 1482 (2008)

J.L. Heldmann, A. Colaprete, D. Wooden, E. Asphaug, P.H. Schultz, C.S. Plesko, L. Ong, D. Korycansky, K. Galal, G. Briggs, Lunar Planet. Sci. Conf., Abstract 1898 (2009)

J.L. Heldmann, T. Colaprete, K. Ennico, M. Shirley, D. Wooden [LCROSS Science Team], Lunar Planet. Sci. Conf., Abstract 1015 (2010)

K.R. Housen, R.M. Schmidt, K.A. Holsapple, J. Geophys. Res. (1983). doi:10.1029/JB088iB03p02485

D.G. Korycansky, C.S. Plesko, M. Jutzi, E. Asphaug, A. Colaprete, Meteor. Planet. Sci. 44, 603-620 (2009)

G.P. Kuiper, E.A. Whitaker, R.G. Strom, J.W. Fountain, S.M. Larson, Consolidated Lunar Atlas (University of Arizona Press, Tucson, 1967)

D.J. Lawrence, W.C. Feldman, R.C. Elphic, J.J. Hagerty, S. Maurice, G.W. McKinney, T.H. Prettyman, J. Geophys. Res. 111 (2006). doi:10.1029/2005JE002637

J.L. Margot, D.B. Campbell, R.F. Jurgens, M.A. Slade, Science 284 (1999). doi:10.1126/science.284. 5420.1658

P. McGregor, J. Hart, P. Conroy, L. Pfitzner, G. Bloxham, D. Jones, M. Downing, M. Dawson, P. Young, M. Jarnyk, J. van Harmelen, SPIE 4841, 178 (2002)

K.J. Meech, et al., Science 310 (2005). doi:10.1126/science.1118978

T.W. Murphy, E.G. Adelberger, J.B.R. Battat, L.N. Carey, C.D. Hoyle, P. LeBlanc, E.L. Michelsen, K. Nordtvedt, A.E. Orin, J.D. Strasburg, C.W. Stubbs, H.E. Swanson, E. Williams, Publ. Astron. Soc. Pac. 120 (2008)

R.E. Nather, A.S. Mukadam, Astrophys. J. 605 (2004). doi:10.1086/382676

S. Nozette, C.L. Lichtenberg, P. Spudis, R. Bonner, W. Ort, E. Malaret, M. Robinson, E.M. Shoemaker, Science 274 (1996). doi:10.1126/science.274.5292.1495

D. Paige, et al., Science (2010). doi:10.1126/science.1187726

C.M. Pieters, J.N. Goswami, R.N. Clark, M. Annadurai, J. Boardman, B. Buratti, J.-P. Combe, M.D. Dyar, R. Green, J.W. Head, C. Hibbitts, M. Hicks, P. Isaacson, R. Klima, G. Kramer, S. Kumar, E. Livo, S. Lundeen, E. Malaret, T. McCord, J. Mustard, J. Nettles, N. Petro, C. Runyon, M. Staid, J. Sunshine, L.A. Taylor, S. Tompkins, P. Varanasi, Science 326 (2009). doi:10.1126/science. 1178658

J.T. Rayner, D.W. Toomey, P.M. Onaka, A.J. Denault, W.E. Stahlberger, W.D. Vacca, M.C. Cushing, S. Wang, Publ. Astron. Soc. Pac. 115 (2003)

A.S. Rivkin, E.S. Howell, F. Vilas, L.A. Lebofsky, in Asteroids III (University of Arizona Press, Tucson, 2002), pp. 235-253

A.S. Rivkin, J.K. Davies, J.R. Johnson, S.L. Ellison, D.E. Trilling, R.H. Brown, L.A. Lebofsky, Meteorit. Planet. Sci. 38, 1383-1398 (2003). doi:10.1111/j.1945-5100.2003.tb00321.x

T. Roman, D. Skillman, User Information Report U.I.R.-2007-001, Space Telescope Science Institute (2007)

R. Russell, et al., in AMOS Technical Conference (2005), pp. 279-286

P. Schultz, et al., Science (2010). doi:10.1126/science.1187454

V.V. Shuvalov, I.A. Trubetskaya, Solar Syst. Res. 42 (2008). doi:10.1134/S0038094608010012

M.A. Skinner, et al., in AMOS Technical Conference (2007)

M.A. Skinner et al., in International Astronomical Congress 60th Meeting, IAC-09-A6.1.17 (2009)

N.J.S. Stacy, D.B. Campbell, P.G. Ford, Science 276 (1997). doi:10.1126/science.276.5318.1527

A.D. Storrs, A. Colaprete, Lunar. Planet. Sci. Conf., Abstract 2196 (2010)

R.M. Suggs, W. Cooke, R. Suggs, H. McNamara, W. Swift, D. Moser, D. Diekmann, Bull. Am. Astron. Soc. 40 (2008a)

R.M. Suggs, W.J. Cooke, R.J. Suggs, W.R. Swift, N. Hollon, Earth, Moon, Planets 102 (2008b). doi:10.1007/s11038-007-9184-0

D. Summy, D. Goldstein, A. Colaprete, P.L. Varghese, L.M. Trafton, Lunar. Planet. Sci. Conf., Abstract 2267 (2009)

J.M. Sunshine, T.L. Farnham, L.M. Feaga, O. Groussin, F. Merlin, R.E. Milliken, M.F. A'Hearn, Science 326 (2009). doi:10.1126/science.1179788

J.E.H. Turner, B.W. Miller, T.L. Beck, I. Song, A.J. Cooke, R.L. Seaman, F.G. Valdes, New Astron. Rev. 49, 655 (2006)

A.R. Vasavada, D.A. Paige, S.E. Wood, Icarus 141 (1999). doi:10.1006/icar.1999.6175

F. Vilas, E.A. Jensen, D.L. Domingue, L.A. McFadden, C. Runyon, W. Mendell, Earth, Planets, Space 60, 67-74 (2008)

F. Vilas, MMT Observatory 6.5m CCD47 Raw Observations of LCROSS, NASA Planetary Data System, EAR-L-MMTO_CCD47-2-EDR-LCROSS-V1.0 (2010a) 
F. Vilas, MMT Observatory 6.5m CLIO Raw Observations of LCROSS, NASA Planetary Data System, EAR-L-MMTO_CLIO-2-EDR-LCROSS-V1.0 (2010b)

F. Vilas, MMT Observatory 6.5m CLIO Calibrated Observations of LCROSS, NASA Planetary Data System, EAR-L-MMTO_CLIO-3-CDR-LCROSS-V1.0 (2010c)

R. Vondrak, J. Keller, G. Chin, J. Garvin, Space Sci. Rev. 150 (2010). doi:10.1007/s11214-010-9631-5 